\title{
On Line Computation of Process Capability Indices
}

\author{
J. Subramani ${ }^{1, *}$, S. Balamurali ${ }^{2}$ \\ ${ }^{1}$ Department of Statistics, Pondicherry University, R V Nagar, Kalapet, Puducherry, 605 014, India \\ ${ }^{2}$ Department of Computer App lications, Kalasalin gam University, Krishnankoil, 626 190, Tamilnadu, India
}

\begin{abstract}
Statistical process control tools and techniques are widely used in assessing and monitoring manufacturing process. Effective implementation of statistical process control tools will improve the productivity and quality; reduce the wastage and improve the business together with various other benefits. Process capability indices are very well used to assess the performance of the production process. However the problem is to keep the produced items in the inventory until to take a decision on the acceptance of the lots based on the outcome of the process capability analysis. An attempt has been made in this paper to overcome such difficulties. As a result we have presented a simple method to assess the process capability indices on line itself, which helps the process as well as the quality engineers to take an instant decision on the manufacturing process whether the process has to be allowed further or to be modified. The method is explained with the help of illustrations.
\end{abstract}

Keywords Manufacturing Range, on Line Computation, Process Capability Index, Samp le Size, Tolerance

\section{Introduction}

The present scenario of total quality management demands the effective use of statistical tools for analysing quality problems and controlling, monitoring, maintaining and improving the performance of manufacturing process. Many of the quality characteristics are measurable in nature and can be expressed in terms of numerical measurements and are to be monitored and controlled during the production. When dealing with measurable quality characteristics, it is usually necess ary to monitor the behaviour of the mean value of the quality characteristics and its variability. To study the behaviour of the production process and to take a necessary action on the process, there are several statistical tools available which include frequency distribution, histogram, Pareto chart, scatter diagram, control charts, regression analysis, process capability analysis, design of experiments and Taguchi methods and are to be effectively used for further improvements on quality and productivity. Among the statistical tools mentioned above, the use of process capability analys is has been increasingly recognised by industrial sectors, particularly the automobile manufacturing industries. The world giant manufacturer of automobiles namely Ford, Chrysler, and General Motors have together developed a Quality System namely QS 9000 standards and advised their suppliers to follow the QS 9000 - Quality Systems Requirements and to get certified for the same to

* Corresponding author:

drjsubramani@yahoo.co.in (J. Subramani)

Published online at http://journal.sapub.org/statistics

Copyright (C) 2012 Scientific \& Academic Publishing. All Rights Reserved continue as one of the potential suppliers to the above automobile manufacturing giants.

The process capability analysis as discussed by Montgomery[10] is a vital part of an overall quality improvement program. Several PCIs have been developed among which the basic indices are $\mathrm{Cp}, \mathrm{Cpk}$ and $\mathrm{Cpm}$ (refer to Kane[8]; Chan et.a1.[5]). A detailed review of the PCIs can be seen in Kotz and Johnson[9] and Yum and Kim[13]. The statistical data collected for assessing the suitability of the manufacturing processes and or for computing the process capability indices can also be used to:

* Predict how well the process will hold the tolerances

* Assist process engineers in selecting a process

* Assist product engineers in modifying a product

* Assist in establishing the frequency of sampling

* Assess the capability of a new equip ment

* Select competing vendors

* Reduce variations

* Predict the outgoing quality level etc.,

However the problem encountered in the present day manufacturing systems are as follows:

* The process capability ind ices are co mputed only after a considerable period.

* There is no method available to ass ess the capability of the process on line

* It requires frequent interference of the process

* It requires storing the produced items for a considerable period before taking the decision to accept or reject or rework based on the outcome of the process capability study.

* In case of allowing the process before getting the process capability indices, there may be a situation, one has to call back the produced items already passed on.

* It requires $100 \%$ inspection and sorting out of the items 
produced in non capable process

The process capability indices $C p, C p k$ and $C p m$ were investigated by many authors. For example, the $95 \%$ confidence limits for the capability indices $C p$ and $C p k$ were constructed by Chou et al.[6]. As their limits on Cpk can produce $97 \%$ or $98 \%$ lower confidence limits (instead of 95\%) making them conservative, an approximation presented by Bissell[3] is recommended. Boyles [4] provided an approximate method for finding lower confidence limits for the index Cpm. All these lower confidence limits are calculated based on the assumption that the process is normally distributed. Franklin and Wasserman[7] constructed the confidence limits for some basic capability indices and examined their behaviours when the underlying process is either normal, skewed or heavy tailed. Balamurali and Kalyanasundaram[2] constructed bootstrap confidence limits for the capability indices $C p, C p k$ and $C p m$ based on lognormal and chi-squared distributions. Balamurali[1] considered the above capability indices under short run production processes and also constructed the bootstrap confidence limits.

An attempt is made in this paper to provide online computation of process capability indices which helps to assess the capability of the process on line itself. As a result a simplified procedure is presented for computing the process capability indices for given sample size, sample range and tolerance instantaneously without actually computing the standard deviations and without using the formulae for computing the process capability indices Consequently we have proposed a new manufacturing system called " Capability Based Manufacturing System", which avoids unnecessary disturbance to the process; $100 \%$ inspection; sorting and segregation; rework and scrap etc. The results are presented in the Table 1 to Table 10 and are given in the appendix. The Tables 1 to 9 can be used to determine the $C_{p}$ value for the given sample size, tole rance and the mean of the sample ranges. On the other hand, it can also be used to determine the manufacturing range to ach ieve the required $C_{p}$ value. Table 10 can be used to determine the $C_{p k}$ value for the given $C_{p}$ and the delta (the proportion of shift in the mean to the targets value). The Table 10 can also be used to know the delta value be allowed to achieve the required $C_{p}$ and $C_{p k}$ values. For want of space we have not presented all the tables developed for various values of $C_{p}$, Tole rance and Delta not included in the Table 1 to Table 10. If anyone interested to get other tables are advised to contact the author by email or post.

\section{Main Results}

The following are the various computational formulae involved in constructing the control charts and for the computation of process capability indices, which will be used to derive the simplified procedure for the computation of online process capability indices:

$$
\begin{aligned}
& \bar{X}_{i}=\frac{1}{n} \sum_{j=1}^{n} X_{i j}: \text { Mean of i-th sample } \\
& \overline{\bar{X}}=\frac{1}{N} \sum_{i=1}^{N} \bar{X}_{i}: \text { Grand Mean } \\
& R_{i}=\operatorname{Max}\left(X_{i 1}, X_{i 2}, X_{i 3}, \ldots, X_{i n}\right)-\operatorname{Min}\left(X_{i 1}, X_{i 2}, X_{i 3}, \ldots, X_{i n}\right):
\end{aligned}
$$

Range of i-th sample

$$
\bar{R}=\frac{1}{k} \sum_{i=1}^{k} R_{i} \quad: \text { Mean of Range values }
$$

$U C L_{\bar{x}}=\overline{\bar{X}}+A_{2} * \bar{R} \quad:$ Upper Control Limit for $\bar{X}$-Chart

$L C L_{\bar{x}}=\overline{\bar{X}}-A_{2} * \bar{R}$ : Lower Control Limit for $\bar{X}$-Chart

$U C L_{R}=D_{4} * \bar{R}$ : Upper Control Limit for R-Chart

$L C L_{R}=D_{3} * \bar{R}$ : Lower Control Limit for R-Chart

$C_{p}=\frac{U S L-L S L}{6 \bar{R} / d_{2}}:$ Process Capability Index

$C_{p u}=\frac{U S L-\overline{\bar{X}}}{3 \bar{R} / d_{2}}:$ Upper Process Capability Index

$C_{p l}=\frac{\overline{\bar{X}}-L S L}{3 \bar{R} / d_{2}}:$ Lower Process Capability Index

$C_{p k}=\operatorname{Min}\left(C_{p u}, C_{p l}\right):$ Process Capability Index

Tolerance $=T=U S L-L S L$

$T \arg e t=M=\frac{U S L+L S L}{2}$

USL: Upper Specification Limit of the Quality Characteristics

LSL: Lower Specification Limit of the Quality Characteristics

For $\bar{X}$ - Chart, the 3-sig ma control limits are obtained as: $\overline{\bar{X}}+3 \sigma_{\bar{X}}$ where $\sigma_{\bar{X}}$ is the standard deviation of sample mean. Further $\sigma_{\bar{X}}=\sigma / \sqrt{n}$, the control limits can be rewritten as $\overline{\bar{X}} \pm 3 \frac{\sigma}{\sqrt{n}}$. Further in Quality Control, the standard deviation $\sigma$ can be normally estimated from the range values using the formula $\sigma=\frac{\bar{R}}{d_{2}}$. Which implies the control limits for the $\bar{X}$-chart using $\bar{R}$ are obtained as $\overline{\bar{X}} \pm 3 \frac{\bar{R}}{d_{2} \sqrt{n}}$, which can be written as $\overline{\bar{X}} \pm A_{2} \bar{R}$ where $A_{2}=\frac{3}{d_{2} \sqrt{n}}$. Similarly, one may obtain the control limits for the R-Charts.

Similarly, two Capability Indices $C_{p}$ and $C_{p k}$ are used for measuring the Process Capability, where $C_{p}$ will take only the variability into consideration and will not consider the centrality of the process for assessing the process capability. Whereas the capability index $C_{p k}$ will take into account both, the variation and centrality of the process. For a detailed discussion on the definition and the derivation of various formulae involved in control charts and process capability analysis one may refer to Montgomery[10], Subraman $[11,12]$ and the references cited there in. 


\subsection{Computation of Aver age Range, Maximum and Minimum Range Values Vs Process Capability Index $C_{p}$ Values}

Consider the process capability index

$$
\begin{array}{r}
C_{p}=\frac{U S L-L S L}{6 \sigma}=\frac{U S L-L S L}{6 \bar{R} / d_{2}} \\
\Rightarrow \bar{R}=\frac{d_{2}(U S L-L S L)}{6 C_{p}}=\frac{d_{2} T}{6 C_{p}},
\end{array}
$$

where $\mathrm{T}$ is the Tolerance.

Further assume that $R_{L}$ and $R_{U}$ are the maximum and the minimum range values in which range the producer can operate the manufacturing process so as to meet the customer requirements including the process capability indices $C_{p}$ and $C_{p k}$ values. That is, the maximum allowable range value should be in between $R_{L}$ and $R_{U}$. The limit of these range values can be obtained from the $\bar{R}$ values obtained in the above equation as follows:

$$
\begin{aligned}
& R_{L}=D_{3} \times \bar{R} \\
& R_{U}=D_{4} \times \bar{R}
\end{aligned}
$$

From the above equations one can easily determine the required average range value and the maximum and minimum values to be operated so as to meet the customer requirements. That is, for any given value of $C_{p}$, sample size $\mathrm{n}$ and the manufacturing tolerance $\mathrm{T}$, as per the requirements of the customer the producer can determine the range values in which he has to operate the manufacturing process. For want of space and for the sake of convenience we have tabulated $R_{L}, \bar{R}$ and $R_{U}$ values for the given values of $2 \leq n \leq 10 \quad$ with increments of $1, \quad 5 \leq T \leq 100 \quad$ with increments of 5 and $1.0 \leq C_{p} \leq 2.0$ with the increments of 0.1 respectively the sample size, tolerance and process capability indexvalues in Tables 1 to 9 . These tables can also be used to determine the value of process capability index $C_{p}$ for the range values, average range in which the process is operating and the sample size by looking at the appropriate columns corresponding to the tolerance and range values by not actually computing the process capability index. This is the advantageous of this study. The above can be explained with the help of the following illustrations.

Suppose that the customer wants to get products produced in a manufacturing process with tolerance 20 and process capability index $C_{p}$ with 1.7. Let us assume that the producer measures the process capability with a sample size of 5 then we use the Table 4 to determine the manufacturing range values. If we look at the Table 4 for $n=5$, it gives that for the given sample size 5 , the tolerance 20 and $C_{p}$ value 1.7 the average range value is 4.56 and the upper value of the range is 9.65 . Hence to achieve the customer needs, the producer has to operate the manufacturing process with manufacturing range of 0 to 9.65 units.

On the other hand the Tables from 1 to 9 can also be used to determine the process capability index $C_{p}$ without actually computing the $\sigma$ value and $C_{p}$ value by using the formulae as detailed below: Let the given specification be $\mathrm{n}=5, \mathrm{~T}=50$ and the operating Range of $\bar{R}$ value is 20 . By looking at the Table 4 with sample size $n=5$, in the row of $\mathrm{T}=50$ and inside the Row the value close to 20 we obtain the $C_{p}$ value must be in between 1 and 1.1 .

\subsection{Computation of Process Capability Index $C_{p k}$ for the shift in the Process Average Vs Process Capability Index $C_{p}$ Values}

Consider the capability index $C_{p k}$ is defined as $C_{p k}=$ Minimum of $\left\{C_{p u}, C_{p l}\right\}$,

Where $C_{p u}=\frac{U S L-\overline{\bar{X}}}{3 \sigma}=\frac{U S L-\overline{\bar{X}}}{3 \bar{R} / d_{2}}$

and $C_{p l}=\frac{\overline{\bar{X}}-L S L}{3 \sigma}=\frac{\overline{\bar{X}}-L S L}{3 \bar{R} / d_{2}}$.

Consider $C_{p u}=\frac{\frac{U S L+L S L}{2}-\overline{\bar{X}}+\frac{U S L-L S L}{2}}{3 \sigma}$

$C_{p u}=\frac{\frac{U S L-L S L}{2}-\left(\overline{\bar{X}}-\frac{U S L+L S L}{2}\right)}{3 \sigma}=\frac{\frac{T}{2}-(\overline{\bar{X}}-M)}{3 \sigma}$

$C_{p u}=\frac{\frac{T}{2}-(\overline{\bar{X}}-M)}{3 \bar{R} / d_{2}}$

Consider

$C_{p l}=\frac{\overline{\bar{X}}-L S L}{3 \sigma}=\frac{\overline{\bar{X}}-L S L}{3 \bar{R} / d_{2}}$

$C_{p l}=\frac{\frac{U S L-L S L}{2}+\overline{\bar{X}}-\frac{U S L+L S L}{2}}{3 \sigma}$

$C_{p l}=\frac{\frac{U S L-L S L}{2}+\left(\overline{\bar{X}}-\frac{U S L+L S L}{2}\right)}{3 \sigma}=\frac{\frac{T}{2}+(\overline{\bar{X}}-M)}{3 \sigma}$

$C_{p l}=\frac{\frac{T}{2}+(\overline{\bar{X}}-M)}{3 \bar{R} / d_{2}}$

Fro $m$ the above expressions one can obtain

$C_{p k}=$ Minimum of $\left\{C_{p u}, C_{p l}\right\}=\frac{\frac{T}{2}-\|\overline{\bar{X}}-M\|}{3 \bar{R} / d_{2}}$

$C_{p k}=\frac{T}{6 \bar{R} / d_{2}}-\frac{\Delta}{3 \bar{R} / d_{2}}$

where $\Delta=\|\overline{\bar{X}}-M\|$

$C_{p k}=\frac{T}{6 \bar{R} / d_{2}}\left(1-\frac{2 \Delta}{T}\right)=C_{p}(1-\partial)$,

where $\partial=\frac{2 \Delta}{T}$, the proportion of shift in the process average to

the total tolerance. If we multiply the $\partial$ by 100 one may get the percentage of the shift in the process average to the total tolerance.

For want of space and for the sake of convenience of the readers, we have tabulated $C_{p k}$ values for the given values 
of $\partial$ (in percentage to the total tolerance) in the range $0 \leq \partial \leq 100$ with increments of 2 and the process capability index values in the range $1.0 \leq C_{p} \leq 2.0$ with the increments of 0.1 respectively in Table 10 . The Table 10 can be used to determine the value of process capability index $C_{p k}$ for the delta values (in percentage to the tolerance) and the process capability index $C_{p}$ by looking at the appropriate columns corresponding to the delta values and the $C_{p}$ values by not actually computing the process capability index $C_{p k}$. The above can be explained with the help of the following illustrations.

Table 10 can be used to determine the value of $C_{p k}$ for the given value of $C_{p}$ and the process shift from the target value. For example with the process capability $C_{p}$ in the range of 1.7 with the delta of 30 the process capability index $C_{p k}$ value will be 1.36 only. Similarly the Table 10 can also be used to decide the amount of shift in process average from the target value to maintain the required process capability indices $C_{p}$ and $C_{p k}$ for the given tolerance.

\section{Conclusions}

The present scenario of total quality management requires effective use of statistical tools and techniques for a continual improvement on the production processes. Particularly, the automobile sectors require the use of process capability indices to assess the suitability of the manufacturing process as well as to assess the products quality. After the initial approval of the manufacturing process, the future performance will be assessed in terms of the process capability indices, for which the data are collected for a longer period. In case, the computed process capability indices have not satis fied the required conditions, the manufactured products will be screened and called back for some times. In such situations the decision will affect the smooth flow of the production line and also increase the cost of production due to withdrawal of some products; screening of the production lot; rework, rejection and inventory of some produced items. To avoid all these problems, we have suggested a method to assess the process capability indices while the production process is on. As a result we reduce the inventory, recall, rework and rejection of produced items. All these features have been explained with the help of numerical examples.

\section{APPENDIX}

Table 1. RL, RBAR and RU values for the given $C_{p}$ value and the Sample Size $\mathrm{n}=2$

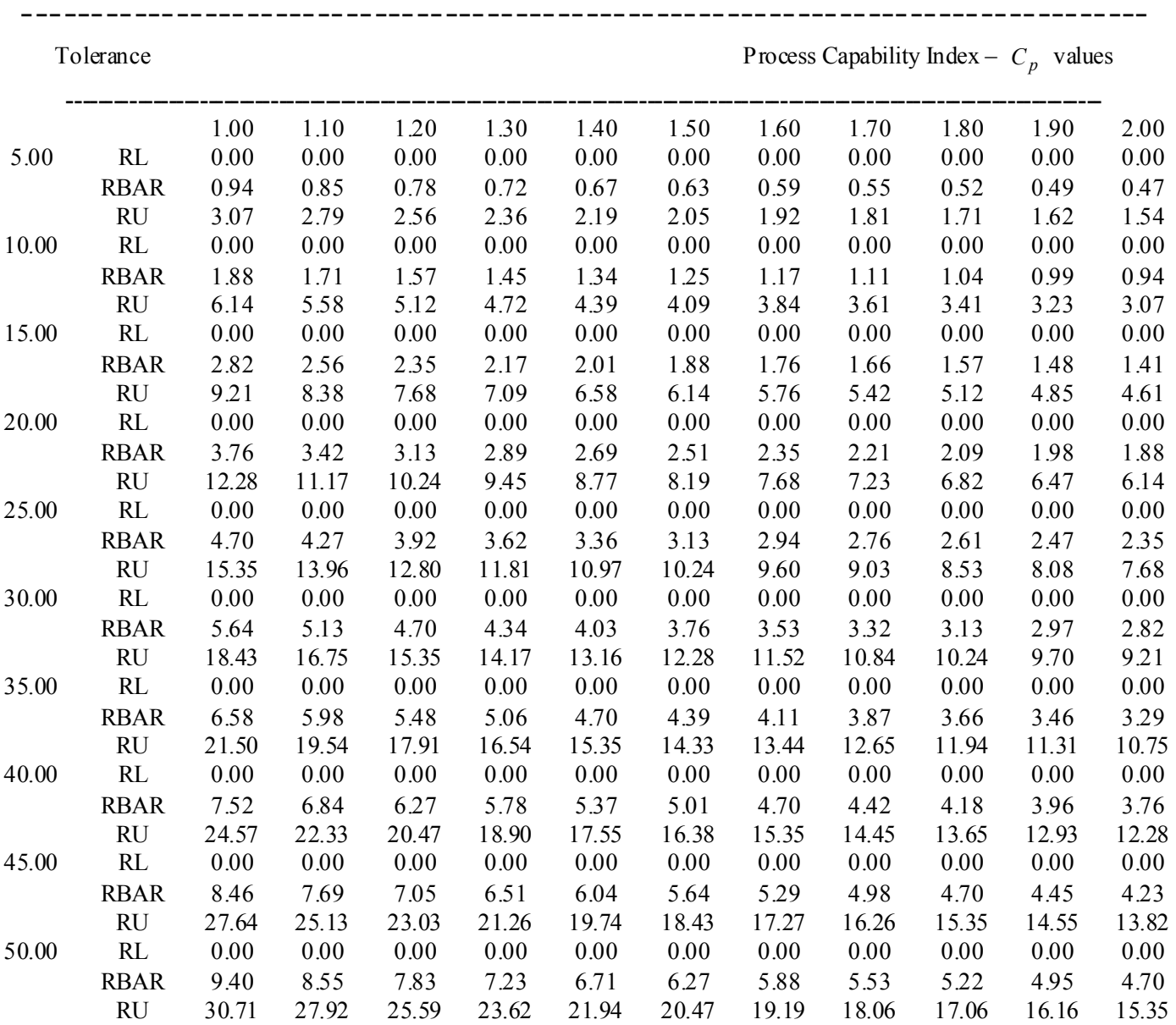




\begin{tabular}{|c|c|c|c|c|c|c|c|c|c|c|c|c|}
\hline \multirow[t]{3}{*}{55.00} & RL & 0.00 & 0.00 & 0.00 & 0.00 & 0.00 & 0.00 & 0.00 & 0.00 & 0.00 & 0.00 & 0.00 \\
\hline & RBAR & 10.34 & 9.40 & 8.62 & 7.95 & 7.39 & 6.89 & 6.46 & 6.08 & 5.74 & 5.44 & 5.17 \\
\hline & RU & 33.78 & 30.71 & 28.15 & 25.99 & 24.13 & 22.52 & 21.11 & 19.87 & 18.77 & 17.78 & 16.89 \\
\hline \multirow[t]{3}{*}{60.00} & RL & 0.00 & 0.00 & 0.00 & 0.00 & 0.00 & 0.00 & 0.00 & 0.00 & 0.00 & 0.00 & 0.00 \\
\hline & RBAR & 11.28 & 10.25 & 9.40 & 8.68 & 8.06 & 7.52 & 7.05 & 6.64 & 6.27 & 5.94 & 5.64 \\
\hline & RU & 36.85 & 33.50 & 30.71 & 28.35 & 26.32 & 24.57 & 23.03 & 21.68 & 20.47 & 19.40 & 18.43 \\
\hline \multirow[t]{3}{*}{65.00} & RL & 0.00 & 0.00 & 0.00 & 0.00 & 0.00 & 0.00 & 0.00 & 0.00 & 0.00 & 0.00 & 0.00 \\
\hline & RBAR & 12.22 & 11.11 & 10.18 & 9.40 & 8.73 & 8.15 & 7.64 & 7.19 & 6.79 & 6.43 & 6.11 \\
\hline & RU & 39.92 & 36.29 & 33.27 & 30.71 & 28.52 & 26.62 & 24.95 & 23.48 & 22.18 & 21.01 & 19.96 \\
\hline \multirow[t]{3}{*}{70.00} & RL & 0.00 & 0.00 & 0.00 & 0.00 & 0.00 & 0.00 & 0.00 & 0.00 & 0.00 & 0.00 & 0.00 \\
\hline & RBAR & 13.16 & 11.96 & 10.97 & 10.12 & 9.40 & 8.77 & 8.23 & 7.74 & 7.31 & 6.93 & 6.58 \\
\hline & RU & 42.99 & 39.09 & 35.83 & 33.07 & 30.71 & 28.66 & 26.87 & 25.29 & 23.89 & 22.63 & 21.50 \\
\hline \multirow[t]{3}{*}{75.00} & RL & 0.00 & 0.00 & 0.00 & 0.00 & 0.00 & 0.00 & 0.00 & 0.00 & 0.00 & 0.00 & 0.00 \\
\hline & RBAR & 14.10 & 12.82 & 11.75 & 10.85 & 10.07 & 9.40 & 8.81 & 8.29 & 7.83 & 7.42 & 7.05 \\
\hline & RU & 46.06 & 41.88 & 38.39 & 35.43 & 32.90 & 30.71 & 28.79 & 27.10 & 25.59 & 24.24 & 23.03 \\
\hline \multirow[t]{3}{*}{80.00} & $\mathrm{RL}$ & 0.00 & 0.00 & 0.00 & 0.00 & 0.00 & 0.00 & 0.00 & 0.00 & 0.00 & 0.00 & 0.00 \\
\hline & RBAR & 15.04 & 13.67 & 12.53 & 11.57 & 10.74 & 10.03 & 9.40 & 8.85 & 8.36 & 7.92 & 7.52 \\
\hline & RU & 49.14 & 44.67 & 40.95 & 37.80 & 35.10 & 32.76 & 30.71 & 28.90 & 27.30 & 25.86 & 24.57 \\
\hline \multirow[t]{3}{*}{85.00} & RL & 0.00 & 0.00 & 0.00 & 0.00 & 0.00 & 0.00 & 0.00 & 0.00 & 0.00 & 0.00 & 0.00 \\
\hline & RBAR & 15.98 & 14.53 & 13.32 & 12.29 & 11.41 & 10.65 & 9.99 & 9.40 & 8.88 & 8.41 & 7.99 \\
\hline & RU & 52.21 & 47.46 & 43.51 & 40.16 & 37.29 & 34.80 & 32.63 & 30.71 & 29.00 & 27.48 & 26.10 \\
\hline \multirow[t]{3}{*}{90.00} & $\mathrm{RL}$ & 0.00 & 0.00 & 0.00 & 0.00 & 0.00 & 0.00 & 0.00 & 0.00 & 0.00 & 0.00 & 0.00 \\
\hline & RBAR & 16.92 & 15.38 & 14.10 & 13.02 & 12.09 & 11.28 & 10.57 & 9.95 & 9.40 & 8.91 & 8.46 \\
\hline & RU & 55.28 & 50.25 & 46.06 & 42.52 & 39.48 & 36.85 & 34.55 & 32.52 & 30.71 & 29.09 & 27.64 \\
\hline \multirow[t]{3}{*}{95.00} & $\mathrm{RL}$ & 0.00 & 0.00 & 0.00 & 0.00 & 0.00 & 0.00 & 0.00 & 0.00 & 0.00 & 0.00 & 0.00 \\
\hline & RBAR & 17.86 & 16.24 & 14.88 & 13.74 & 12.76 & 11.91 & 11.16 & 10.51 & 9.92 & 9.40 & 8.93 \\
\hline & RU & 58.35 & 53.04 & 48.62 & 44.88 & 41.68 & 38.90 & 36.47 & 34.32 & 32.42 & 30.71 & 29.17 \\
\hline \multirow[t]{3}{*}{100.00} & RL & 0.00 & 0.00 & 0.00 & 0.00 & 0.00 & 0.00 & 0.00 & 0.00 & 0.00 & 0.00 & 0.00 \\
\hline & RBAR & 18.80 & 17.09 & 15.67 & 14.46 & 13.43 & 12.53 & 11.75 & 11.06 & 10.44 & 9.89 & 9.40 \\
\hline & RU & 61.42 & 55.84 & 51.18 & 47.25 & 43.87 & 40.95 & 38.39 & 36.13 & 34.12 & 32.33 & 30.71 \\
\hline
\end{tabular}

Table 2. RL, RBAR and RU values for the given $C_{p}$ value and the Sample Size $\mathrm{n}=3$

Tolerance

\begin{tabular}{|c|c|c|c|c|c|c|c|c|c|c|c|c|}
\hline & & 1.00 & 1.10 & 1.20 & 1.30 & 1.40 & 1.50 & 1.60 & 1.70 & 1.80 & 1.90 & 2.00 \\
\hline \multirow[t]{3}{*}{5.00} & $\mathrm{RL}$ & 0.00 & 0.00 & 0.00 & 0.00 & 0.00 & 0.00 & 0.00 & 0.00 & 0.00 & .00 & 0.00 \\
\hline & RBAR & 1.41 & 1.28 & 1.18 & 1.09 & 1.01 & 0.94 & 0.88 & 0.83 & .78 & .74 & .71 \\
\hline & RU & 3.63 & 3.30 & 3.03 & 2.79 & 2.59 & 42 & 2.27 & 2.14 & .02 & .91 & .82 \\
\hline \multirow[t]{3}{*}{10.00} & $\mathrm{RL}$ & 0.00 & 0.00 & 0.00 & 0.00 & 0.00 & 00 & 0.00 & 0.00 & .00 & .00 & .00 \\
\hline & RBAR & 2.82 & 2.57 & 2.35 & 2.17 & 2.02 & 88 & & & .57 & .49 & .41 \\
\hline & RU & 7.26 & 6.60 & 6.05 & & & & & & & & .63 \\
\hline \multirow[t]{3}{*}{15.00} & RL & 0.00 & 0.00 & 0.00 & & & & & & & & .00 \\
\hline & RBAR & 4.23 & 3.85 & 3.53 & 3.26 & 3.02 & & 2.65 & 2.4 & 2.35 & 3 & 2.12 \\
\hline & RU & 10.89 & 9.9 & & & & & & & & & .45 \\
\hline \multirow[t]{3}{*}{20.00} & RL & 0.00 & 0.00 & 0.00 & & & & 0.0 & 0.0 & & & .00 \\
\hline & RBAR & 5.64 & 5.13 & 4.70 & 4.34 & 4.03 & 3.76 & 3.53 & 3.32 & 4 & & 2.82 \\
\hline & RU & 14.53 & 13.21 & 12.10 & 11.1 & 10.38 & & 9.08 & & & & .26 \\
\hline \multirow[t]{3}{*}{25.00} & RL & & 0.00 & 0.00 & 0.00 & & & & & & & 0.00 \\
\hline & RBAR & 7.05 & 6.41 & 5.88 & 5.43 & 5.0 & 4.7 & 4.41 & 4.15 & 3.92 & & 3.53 \\
\hline & & 18.16 & 16. & 15.1 & 13.9 & 12. & 12. & 11.35 & 10.68 & 10. & & 9.08 \\
\hline \multirow[t]{3}{*}{30.00} & $\mathrm{RL}$ & 0.0 & 0.0 & 0.0 & 0.0 & 0.0 & 0.0 & 0.00 & 0.00 & 0.0 & .00 & 0.00 \\
\hline & RBAR & 8.4 & 7.7 & 7.0 & 6.5 & 6.0 & 5.6 & 5.2 & 4.9 & 4.7 & 6 & 4.23 \\
\hline & RU & 21.7 & 19.8 & 18.1 & $16^{\circ}$ & 15. & 14. & & 12.82 & 12. & & 10.89 \\
\hline \multirow[t]{3}{*}{35.00} & RL & & 0.0 & & 0. & 0.0 & 0.0 & 0.0 & 0.0 & 0.0 & 0.0 & 0.00 \\
\hline & RBAR & & 8.9 & 8.2 & 7.6 & & 6.5 & 6.1 & 5.8 & 5.4 & 5.2 & 4.94 \\
\hline & RU & 25.42 & 23.11 & 21.18 & 19.5 & 18.16 & 16. & 15.89 & 14.95 & 14. & & 12.71 \\
\hline \multirow[t]{3}{*}{40.00} & RL & & 0.00 & 0.00 & 0.00 & & 0.0 & 0.00 & 0.0 & 0.0 & & 0.00 \\
\hline & RBAR & 11.29 & 10.26 & 9.41 & 8.68 & 8.06 & 7.52 & 7.05 & 6.64 & 6.27 & 5.94 & 5.64 \\
\hline & RU & 29.05 & 26.41 & 24.21 & 22.35 & 20.75 & 19.37 & 18.16 & 17.09 & 16.14 & 15.29 & 14.53 \\
\hline \multirow[t]{3}{*}{45.00} & $\mathrm{RL}$ & 0.00 & 0.00 & 0.00 & 0.00 & 0.00 & 0.00 & 0.00 & 0.00 & 0.00 & 0.00 & 0.00 \\
\hline & RBAR & 12.70 & 11.54 & 10.58 & 9.77 & 9.07 & 8.47 & 7.94 & 7.47 & 7.05 & 6.68 & 6.35 \\
\hline & RU & 32.68 & 29.71 & 27.24 & 25.14 & 23.35 & 21.79 & 20.43 & & 18.16 & 17.20 & 16.34 \\
\hline \multirow[t]{2}{*}{50.00} & $\mathrm{RL}$ & 0.00 & 0.00 & 0.00 & 0.00 & 0.00 & 0.00 & 0.00 & 0.00 & 0.00 & 0.00 & 0.00 \\
\hline & RBAR & 14.11 & 12.83 & 11.76 & 10.85 & 10.08 & 9.41 & 8.82 & 8.30 & 7.84 & 7.43 & 7.05 \\
\hline
\end{tabular}




\begin{tabular}{|c|c|c|c|c|c|c|c|c|c|c|c|c|}
\hline \multirow{4}{*}{55.00} & $\mathrm{RU}$ & 36.31 & 33.01 & 30.26 & 27.93 & 25.94 & 24.21 & 22.70 & 21.36 & 20.17 & 19.11 & 18.16 \\
\hline & $\mathrm{RL}$ & 0.00 & 0.00 & 0.00 & 0.00 & 0.00 & 0.00 & 0.00 & 0.00 & 0.00 & 0.00 & 0.00 \\
\hline & RBAR & 15.52 & 14.11 & 12.93 & 11.94 & 11.09 & 10.35 & 9.70 & 9.13 & 8.62 & 8.17 & 7.76 \\
\hline & RU & 39.95 & 36.31 & 33.29 & 30.73 & 28.53 & 26.63 & 24.97 & 23.50 & 22.19 & 21.02 & 19.97 \\
\hline \multirow[t]{3}{*}{60.00} & RL & 0.00 & 0.00 & 0.00 & 0.00 & 0.00 & 0.00 & 0.00 & 0.00 & 0.00 & 0.00 & 0.00 \\
\hline & RBAR & 16.93 & 15.39 & 14.11 & 13.02 & 12.09 & 11.29 & 10.58 & 9.96 & 9.41 & 8.91 & 8.47 \\
\hline & RU & 43.58 & 39.62 & 36.31 & 33.52 & 31.13 & 29.05 & 27.24 & 25.63 & 24.21 & 22.94 & 21.79 \\
\hline \multirow[t]{3}{*}{65.00} & $\mathrm{RL}$ & 0.00 & 0.00 & 0.00 & 0.00 & 0.00 & 0.00 & 0.00 & 0.00 & 0.00 & 0.00 & 0.00 \\
\hline & RBAR & 18.34 & 16.67 & 15.28 & 14.11 & 13.10 & 12.23 & 11.46 & 10.79 & 10.19 & 9.65 & 9.17 \\
\hline & RU & 47.21 & 42.92 & 39.34 & 36.31 & 33.72 & 31.47 & 29.51 & 27.77 & 26.23 & 24.85 & 23.60 \\
\hline \multirow[t]{3}{*}{70.00} & RL & 0.00 & 0.00 & 0.00 & 0.00 & 0.00 & 0.00 & 0.00 & 0.00 & 0.00 & 0.00 & 0.00 \\
\hline & RBAR & 19.75 & 17.96 & 16.46 & 15.19 & 14.11 & 13.17 & 12.34 & 11.62 & 10.97 & 10.40 & 9.88 \\
\hline & RU & 50.84 & 46.22 & 42.37 & 39.11 & 36.31 & 33.89 & 31.78 & 29.91 & 28.24 & 26.76 & 25.42 \\
\hline \multirow[t]{3}{*}{75.00} & $\mathrm{RL}$ & 0.00 & 0.00 & 0.00 & 0.00 & 0.00 & 0.00 & 0.00 & 0.00 & 0.00 & 0.00 & 0.00 \\
\hline & RBAR & 21.16 & 19.24 & 17.64 & 16.28 & 15.12 & 14.11 & 13.23 & 12.45 & 11.76 & 11.14 & 10.58 \\
\hline & RU & 54.47 & 49.52 & 45.39 & 41.90 & 38.91 & 36.31 & 34.05 & 32.04 & 30.26 & 28.67 & 27.24 \\
\hline \multirow[t]{3}{*}{80.00} & $\mathrm{RL}$ & 0.00 & 0.00 & 0.00 & 0.00 & 0.00 & 0.00 & 0.00 & 0.00 & 0.00 & 0.00 & 0.00 \\
\hline & RBAR & 22.57 & 20.52 & 18.81 & 17.36 & 16.12 & 15.05 & 14.11 & 13.28 & 12.54 & 11.88 & 11.29 \\
\hline & RU & 58.10 & 52.82 & 48.42 & 44.70 & 41.50 & 38.74 & 36.31 & 34.18 & 32.28 & 30.58 & 29.05 \\
\hline \multirow[t]{3}{*}{85.00} & $\mathrm{RL}$ & 0.00 & 0.00 & 0.00 & 0.00 & 0.00 & 0.00 & 0.00 & 0.00 & 0.00 & 0.00 & 0.00 \\
\hline & RBAR & 23.98 & 21.80 & 19.99 & 18.45 & 17.13 & 15.99 & 14.99 & 14.11 & 13.32 & 12.62 & 11.99 \\
\hline & RU & 61.74 & 56.12 & 51.45 & 47.49 & 44.10 & 41.16 & 38.58 & 36.31 & 34.30 & 32.49 & 30.87 \\
\hline \multirow[t]{3}{*}{90.00} & RL & 0.00 & 0.00 & 0.00 & 0.00 & 0.00 & 0.00 & 0.00 & 0.00 & 0.00 & 0.00 & 0.00 \\
\hline & RBAR & 25.39 & 23.09 & 21.16 & 19.53 & 18.14 & 16.93 & 15.87 & 14.94 & 14.11 & 13.37 & 12.70 \\
\hline & RU & 65.37 & 59.42 & 54.47 & 50.28 & 46.69 & 43.58 & 40.85 & 38.45 & 36.31 & 34.40 & 32.68 \\
\hline \multirow[t]{3}{*}{95.00} & $\mathrm{RL}$ & 0.00 & 0.00 & 0.00 & 0.00 & 0.00 & 0.00 & 0.00 & 0.00 & 0.00 & 0.00 & 0.00 \\
\hline & RBAR & 26.81 & 24.37 & 22.34 & 20.62 & 19.15 & 17.87 & 16.75 & 15.77 & 14.89 & 14.11 & 13.40 \\
\hline & RU & 69.00 & 62.73 & 57.50 & 53.08 & 49.28 & 46.00 & 43.12 & 40.59 & 38.33 & 36.31 & 34.50 \\
\hline \multirow[t]{3}{*}{100.00} & RL & 0.00 & 0.00 & 0.00 & 0.00 & 0.00 & 0.00 & 0.00 & 0.00 & 0.00 & 0.00 & 0.00 \\
\hline & RBAR & 28.22 & 25.65 & 23.51 & 21.71 & 20.15 & 18.81 & 17.64 & 16.60 & 15.68 & 14.85 & 14.11 \\
\hline & RU & 72.63 & 66.03 & 60.52 & 55.87 & 51.88 & 48.42 & 45.39 & 42.72 & 40.35 & 38.23 & 36.31 \\
\hline
\end{tabular}

Table 3. RL, RBAR and RU values for the given $C_{p}$ value and the Sample Size $\mathrm{n}=4$

Tolerance

Process Capability Index $-C_{p}$ values

\begin{tabular}{|c|c|c|c|c|c|c|c|c|c|c|c|c|}
\hline & & 1.00 & 1.10 & 1.20 & 1.30 & 1.40 & 1.50 & 1.60 & 1.70 & 1.80 & 1.90 & 2.00 \\
\hline \multirow[t]{3}{*}{5.00} & RL & & & & & & & & & & & 0.00 \\
\hline & RBAR & 1.72 & 1.56 & & 1.32 & 1.23 & & & & 0.95 & 0.90 & 0.86 \\
\hline & RU & 3.92 & & & & & & & & 2.18 & 2.06 & 1.96 \\
\hline \multirow[t]{3}{*}{10.00} & RL & .00 & 0.00 & & 0.00 & & & & & & 0.00 & 0.00 \\
\hline & RBAR & .43 & 3.12 & & 2.64 & & & & & & & 1.72 \\
\hline & RU & & & & & & & & & & & 3.92 \\
\hline \multirow[t]{3}{*}{15.00} & RL & & & & & & & & & & & 0.00 \\
\hline & & & & & & & & & & & & 2.57 \\
\hline & RU & 1 & 10.6 & & 9.0 & & 7 & & & & & 5.87 \\
\hline \multirow[t]{3}{*}{20.00} & RL & & & & & & & & & & & 0.00 \\
\hline & RBA & & & & & & & & & & & 3.43 \\
\hline & & & & & 12. & & & & & & & 7.83 \\
\hline \multirow[t]{3}{*}{25.00} & RL & & & & & & & & & & & 0.00 \\
\hline & RBAR & & & & & & & & & & & 4.29 \\
\hline & & 19.58 & 17.80 & 16.31 & 15.06 & 13.98 & 13.05 & & & 10.88 & 10.30 & 9.79 \\
\hline \multirow[t]{3}{*}{30.00} & & & & & & & & & & & & 0.00 \\
\hline & RBAR & 1 & 9.3 & & 7.9 & 7.3 & 6.8 & & & & & 5.15 \\
\hline & RU & 23.49 & 21.36 & 19.58 & 18.07 & 16.78 & 15.66 & 14.68 & 13.82 & 13.05 & 12.36 & 11.75 \\
\hline \multirow[t]{3}{*}{35.00} & & & & & & & & & & & & 0.00 \\
\hline & RBAR & & & & & & & & & & & 6.01 \\
\hline & RU & 27.41 & 24.92 & 22.84 & 21.08 & 19.58 & 18.27 & 17.13 & 16.12 & 15.23 & 14.43 & 13.70 \\
\hline \multirow[t]{3}{*}{40.00} & RL & & & & & & & & & & & 0.00 \\
\hline & & & & & & & & & & & & 6.86 \\
\hline & & & & & & & & & & & & 15.66 \\
\hline \multirow[t]{3}{*}{45.00} & & & & & & & & & & & & 0.00 \\
\hline & RBAR & & & & & & & & & & & 7.72 \\
\hline & RU & 35.24 & 32.04 & 29.37 & 27.11 & 25.17 & 23.49 & 22.02 & 20.73 & 19.58 & 18.55 & 17.62 \\
\hline \multirow[t]{2}{*}{50.00} & $\mathrm{RL}$ & 0.00 & 0.00 & & 0.00 & 0.00 & 0.00 & 0.00 & 0.00 & 0.00 & 0.00 & 0.00 \\
\hline & RBAR & 17.16 & 15.60 & 14.30 & 13.20 & 12.26 & 11.44 & 10.72 & 10.09 & 9.53 & 9.03 & 8.58 \\
\hline
\end{tabular}




\begin{tabular}{|c|c|c|c|c|c|c|c|c|c|c|c|c|}
\hline \multirow{3}{*}{55.00} & RU & 39.16 & 35.60 & 32.63 & 30.12 & 27.97 & 26.10 & 24.47 & 23.03 & 21.75 & 20.61 & 19.58 \\
\hline & RL & 0.00 & 0.00 & 0.00 & 0.00 & 0.00 & 0.00 & 0.00 & 0.00 & 0.00 & 0.00 & 0.00 \\
\hline & RBAR & 18.87 & 17.16 & 15.73 & 14.52 & 13.48 & 12.58 & 11.80 & 11.10 & 10.49 & 9.93 & 9.44 \\
\hline & RU & 43.07 & 39.16 & 35.89 & 33.13 & 30.76 & 28.71 & 26.92 & 25.34 & 23.93 & 22.67 & 21.54 \\
\hline \multirow[t]{3}{*}{60.00} & $\mathrm{RL}$ & 0.00 & 0.00 & 0.00 & 0.00 & 0.00 & 0.00 & 0.00 & 0.00 & 0.00 & 0.00 & 0.00 \\
\hline & RBAR & 20.59 & 18.72 & 17.16 & 15.84 & 14.71 & 13.73 & 12.87 & 12.11 & 11.44 & 10.84 & 10.30 \\
\hline & RU & 46.99 & 42.71 & 39.16 & 36.14 & 33.56 & 31.32 & 29.37 & 27.64 & 26.10 & 24.73 & 23.49 \\
\hline \multirow[t]{3}{*}{65.00} & RL & 0.00 & 0.00 & 0.00 & 0.00 & 0.00 & 0.00 & 0.00 & 0.00 & 0.00 & 0.00 & 0.00 \\
\hline & RBAR & 22.31 & 20.28 & 18.59 & 17.16 & 15.93 & 14.87 & 13.94 & 13.12 & 12.39 & 11.74 & 11.15 \\
\hline & RU & 50.90 & 46.27 & 42.42 & 39.16 & 36.36 & 33.93 & 31.81 & 29.94 & 28.28 & 26.79 & 25.45 \\
\hline \multirow[t]{3}{*}{70.00} & RL & 0.00 & 0.00 & 0.00 & 0.00 & 0.00 & 0.00 & 0.00 & 0.00 & 0.00 & 0.00 & 0.00 \\
\hline & RBAR & 24.02 & 21.84 & 20.02 & 18.48 & 17.16 & 16.01 & 15.01 & 14.13 & 13.35 & 12.64 & 12.01 \\
\hline & RU & 54.82 & 49.83 & 45.68 & 42.17 & 39.16 & 36.54 & 34.26 & 32.25 & 30.45 & 28.85 & 27.41 \\
\hline \multirow[t]{3}{*}{75.00} & RL & 0.00 & 0.00 & 0.00 & 0.00 & 0.00 & 0.00 & 0.00 & 0.00 & 0.00 & 0.00 & 0.00 \\
\hline & RBAR & 25.74 & 23.40 & 21.45 & 19.80 & 18.38 & 17.16 & 16.09 & 15.14 & 14.30 & 13.55 & 12.87 \\
\hline & RU & 58.73 & 53.39 & 48.94 & 45.18 & 41.95 & 39.16 & 36.71 & 34.55 & 32.63 & 30.91 & 29.37 \\
\hline \multirow[t]{3}{*}{80.00} & RL & 0.00 & 0.00 & 0.00 & 0.00 & 0.00 & 0.00 & 0.00 & 0.00 & 0.00 & 0.00 & 0.00 \\
\hline & RBAR & 27.45 & 24.96 & 22.88 & 21.12 & 19.61 & 18.30 & 17.16 & 16.15 & 15.25 & 14.45 & 13.73 \\
\hline & RU & 62.65 & 56.95 & 52.21 & 48.19 & 44.75 & 41.77 & 39.16 & 36.85 & 34.80 & 32.97 & 31.32 \\
\hline \multirow[t]{3}{*}{85.00} & RL & 0.00 & 0.00 & 0.00 & 0.00 & 0.00 & 0.00 & 0.00 & 0.00 & 0.00 & 0.00 & 0.00 \\
\hline & RBAR & 29.17 & 26.52 & 24.31 & 22.44 & 20.84 & 19.45 & 18.23 & 17.16 & 16.21 & 15.35 & 14.58 \\
\hline & RU & 66.56 & 60.51 & 55.47 & 51.20 & 47.55 & 44.38 & 41.60 & 39.16 & 36.98 & 35.03 & 33.28 \\
\hline \multirow[t]{3}{*}{90.00} & RL & 0.00 & 0.00 & 0.00 & 0.00 & 0.00 & 0.00 & 0.00 & 0.00 & 0.00 & 0.00 & 0.00 \\
\hline & RBAR & 30.89 & 28.08 & 25.74 & 23.76 & 22.06 & 20.59 & 19.30 & 18.17 & 17.16 & 16.26 & 15.44 \\
\hline & RU & 70.48 & 64.07 & 58.73 & 54.22 & 50.34 & 46.99 & 44.05 & 41.46 & 39.16 & 37.09 & 35.24 \\
\hline \multirow[t]{3}{*}{95.00} & RL & 0.00 & 0.00 & 0.00 & 0.00 & 0.00 & 0.00 & 0.00 & 0.00 & 0.00 & 0.00 & 0.00 \\
\hline & RBAR & 32.60 & 29.64 & 27.17 & 25.08 & 23.29 & 21.73 & 20.38 & 19.18 & 18.11 & 17.16 & 16.30 \\
\hline & RU & 74.40 & 67.63 & 62.00 & 57.23 & 53.14 & 49.60 & 46.50 & 43.76 & 41.33 & 39.16 & 37.20 \\
\hline \multirow[t]{3}{*}{100.00} & RL & 0.00 & 0.00 & 0.00 & 0.00 & 0.00 & 0.00 & 0.00 & 0.00 & 0.00 & 0.00 & 0.00 \\
\hline & RBAR & 34.32 & 31.20 & 28.60 & 26.40 & 24.51 & 22.88 & 21.45 & 20.19 & 19.06 & 18.06 & 17.16 \\
\hline & RU & 78.31 & 71.19 & 65.26 & 60.24 & 55.94 & 52.21 & 48.94 & 46.07 & 43.51 & 41.22 & 39.16 \\
\hline
\end{tabular}

Table 4. RL, RBAR and RU values for the given $C_{p}$ value and the Sample Size $\mathrm{n}=5$

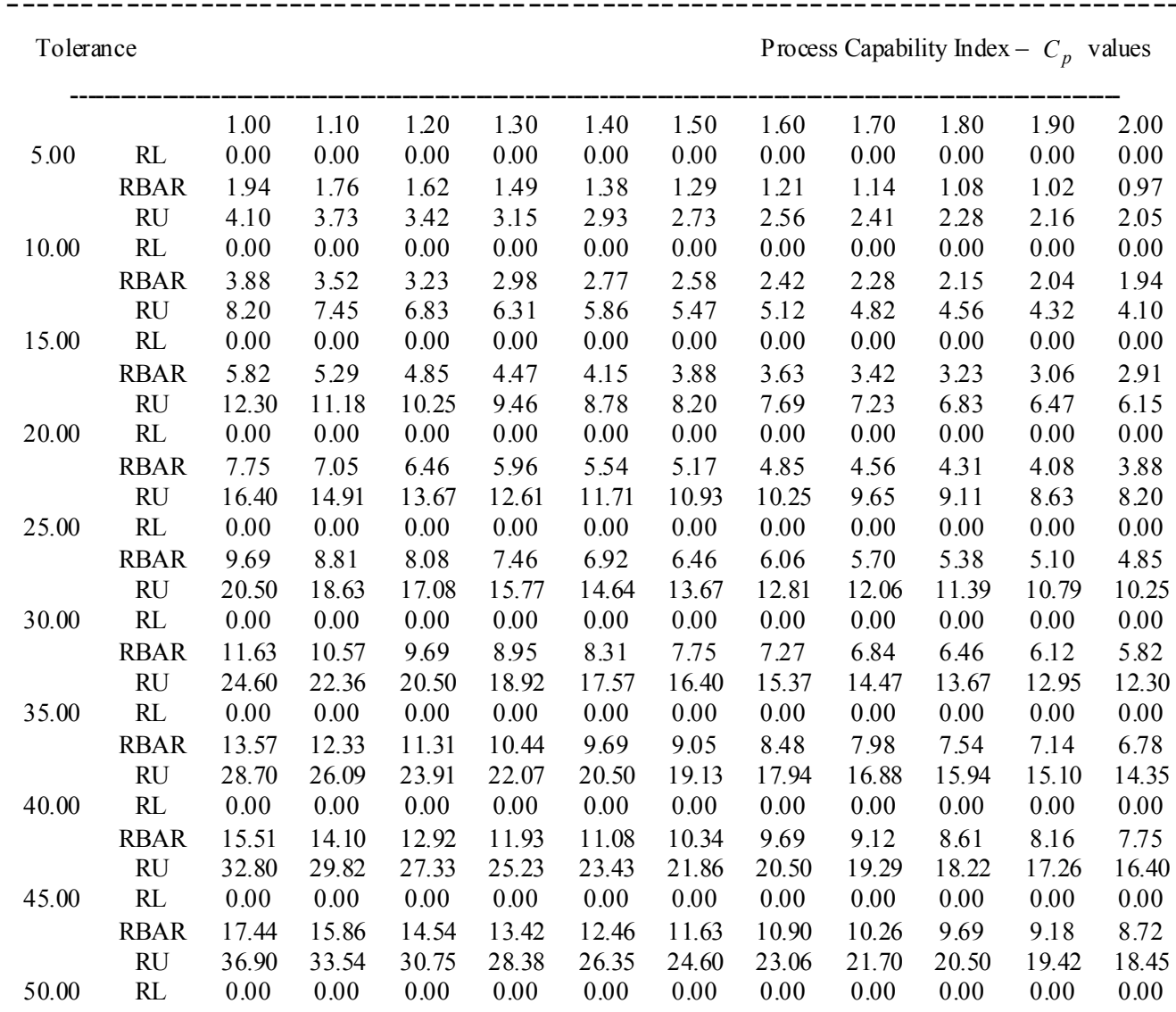




\begin{tabular}{|c|c|c|c|c|c|c|c|c|c|c|c|c|}
\hline & RBAR & 19.38 & 17.62 & 16.15 & 14.91 & 13.85 & 12.92 & 12.11 & 11.40 & 10.77 & 10.20 & 9.69 \\
\hline & RU & 41.00 & 37.27 & 34.16 & 31.54 & 29.28 & 27.33 & 25.62 & 24.12 & 22.78 & 21.58 & 20.50 \\
\hline \multirow[t]{3}{*}{55.00} & RL & 0.00 & 0.00 & 0.00 & 0.00 & 0.00 & 0.00 & 0.00 & 0.00 & 0.00 & 0.00 & 0.00 \\
\hline & RBAR & 21.32 & 19.38 & 17.77 & 16.40 & 15.23 & 14.21 & 13.33 & 12.54 & 11.85 & 11.22 & 10.66 \\
\hline & RU & 45.10 & 41.00 & 37.58 & 34.69 & 32.21 & 30.06 & 28.18 & 26.53 & 25.05 & 23.73 & 22.55 \\
\hline \multirow[t]{3}{*}{60.00} & RL & 0.00 & 0.00 & 0.00 & 0.00 & 0.00 & 0.00 & 0.00 & 0.00 & 0.00 & 0.00 & 0.00 \\
\hline & RBAR & 23.26 & 21.15 & 19.38 & 17.89 & 16.61 & 15.51 & 14.54 & 13.68 & 12.92 & 12.24 & 11.63 \\
\hline & RU & 49.19 & 44.72 & 41.00 & 37.84 & 35.14 & 32.80 & 30.75 & 28.94 & 27.33 & 25.89 & 24.60 \\
\hline \multirow[t]{3}{*}{65.00} & RL & 0.00 & 0.00 & 0.00 & 0.00 & 0.00 & 0.00 & 0.00 & 0.00 & 0.00 & 0.00 & 0.00 \\
\hline & RBAR & 25.20 & 22.91 & 21.00 & 19.38 & 18.00 & 16.80 & 15.75 & 14.82 & 14.00 & 13.26 & 12.60 \\
\hline & RU & 53.29 & 48.45 & 44.41 & 41.00 & 38.07 & 35.53 & 33.31 & 31.35 & 29.61 & 28.05 & 26.65 \\
\hline \multirow{3}{*}{70.00} & RL & 0.00 & 0.00 & 0.00 & 0.00 & 0.00 & 0.00 & 0.00 & 0.00 & 0.00 & 0.00 & 0.00 \\
\hline & RBAR & 27.14 & 24.67 & 22.61 & 20.87 & 19.38 & 18.09 & 16.96 & 15.96 & 15.08 & 14.28 & 13.57 \\
\hline & RU & 57.39 & 52.18 & 47.83 & 44.15 & 41.00 & 38.26 & 35.87 & 33.76 & 31.89 & 30.21 & 28.70 \\
\hline \multirow[t]{3}{*}{75.00} & $\mathrm{RL}$ & 0.00 & 0.00 & 0.00 & 0.00 & 0.00 & 0.00 & 0.00 & 0.00 & 0.00 & 0.00 & 0.00 \\
\hline & RBAR & 29.07 & 26.43 & 24.23 & 22.37 & 20.77 & 19.38 & 18.17 & 17.10 & 16.15 & 15.30 & 14.54 \\
\hline & RU & 61.49 & 55.90 & 51.24 & 47.30 & 43.92 & 41.00 & 38.43 & 36.17 & 34.16 & 32.37 & 30.75 \\
\hline \multirow[t]{3}{*}{80.00} & RL & 0.00 & 0.00 & 0.00 & 0.00 & 0.00 & 0.00 & 0.00 & 0.00 & 0.00 & 0.00 & 0.00 \\
\hline & RBAR & 31.01 & 28.19 & 25.84 & 23.86 & 22.15 & 20.68 & 19.38 & 18.24 & 17.23 & 16.32 & 15.51 \\
\hline & RU & 65.59 & 59.63 & 54.66 & 50.46 & 46.85 & 43.73 & 41.00 & 38.58 & 36.44 & 34.52 & 32.80 \\
\hline \multirow[t]{3}{*}{85.00} & $\mathrm{RL}$ & 0.00 & 0.00 & 0.00 & 0.00 & 0.00 & 0.00 & 0.00 & 0.00 & 0.00 & 0.00 & 0.00 \\
\hline & RBAR & 32.95 & 29.96 & 27.46 & 25.35 & 23.54 & 21.97 & 20.59 & 19.38 & 18.31 & 17.34 & 16.48 \\
\hline & RU & 69.69 & 63.36 & 58.08 & 53.61 & 49.78 & 46.46 & 43.56 & 41.00 & 38.72 & 36.68 & 34.85 \\
\hline \multirow[t]{3}{*}{90.00} & RL & 0.00 & 0.00 & 0.00 & 0.00 & 0.00 & 0.00 & 0.00 & 0.00 & 0.00 & 0.00 & 0.00 \\
\hline & RBAR & 34.89 & 31.72 & 29.08 & 26.84 & 24.92 & 23.26 & 21.81 & 20.52 & 19.38 & 18.36 & 17.44 \\
\hline & RU & 73.79 & 67.08 & 61.49 & 56.76 & 52.71 & 49.19 & 46.12 & 43.41 & 41.00 & 38.84 & 36.90 \\
\hline \multirow[t]{3}{*}{95.00} & RL & 0.00 & 0.00 & 0.00 & 0.00 & 0.00 & 0.00 & 0.00 & 0.00 & 0.00 & 0.00 & 0.00 \\
\hline & RBAR & 36.83 & 33.48 & 30.69 & 28.33 & 26.31 & 24.55 & 23.02 & 21.66 & 20.46 & 19.38 & 18.41 \\
\hline & RU & 77.89 & 70.81 & 64.91 & 59.92 & 55.64 & 51.93 & 48.68 & 45.82 & 43.27 & 41.00 & 38.95 \\
\hline \multirow[t]{3}{*}{100.00} & RL & 0.00 & 0.00 & 0.00 & 0.00 & 0.00 & 0.00 & 0.00 & 0.00 & 0.00 & 0.00 & 0.00 \\
\hline & RBAR & 38.77 & 35.24 & 32.31 & 29.82 & 27.69 & 25.84 & 24.23 & 22.80 & 21.54 & 20.40 & 19.38 \\
\hline & RU & 81.99 & 74.54 & 68.33 & 63.07 & 58.57 & 54.66 & 51.24 & 48.23 & 45.55 & 43.15 & 41.00 \\
\hline
\end{tabular}

Table 5. RL, RBAR and RU values for the given $C_{p}$ value and the Sample Size $\mathrm{n}=6$

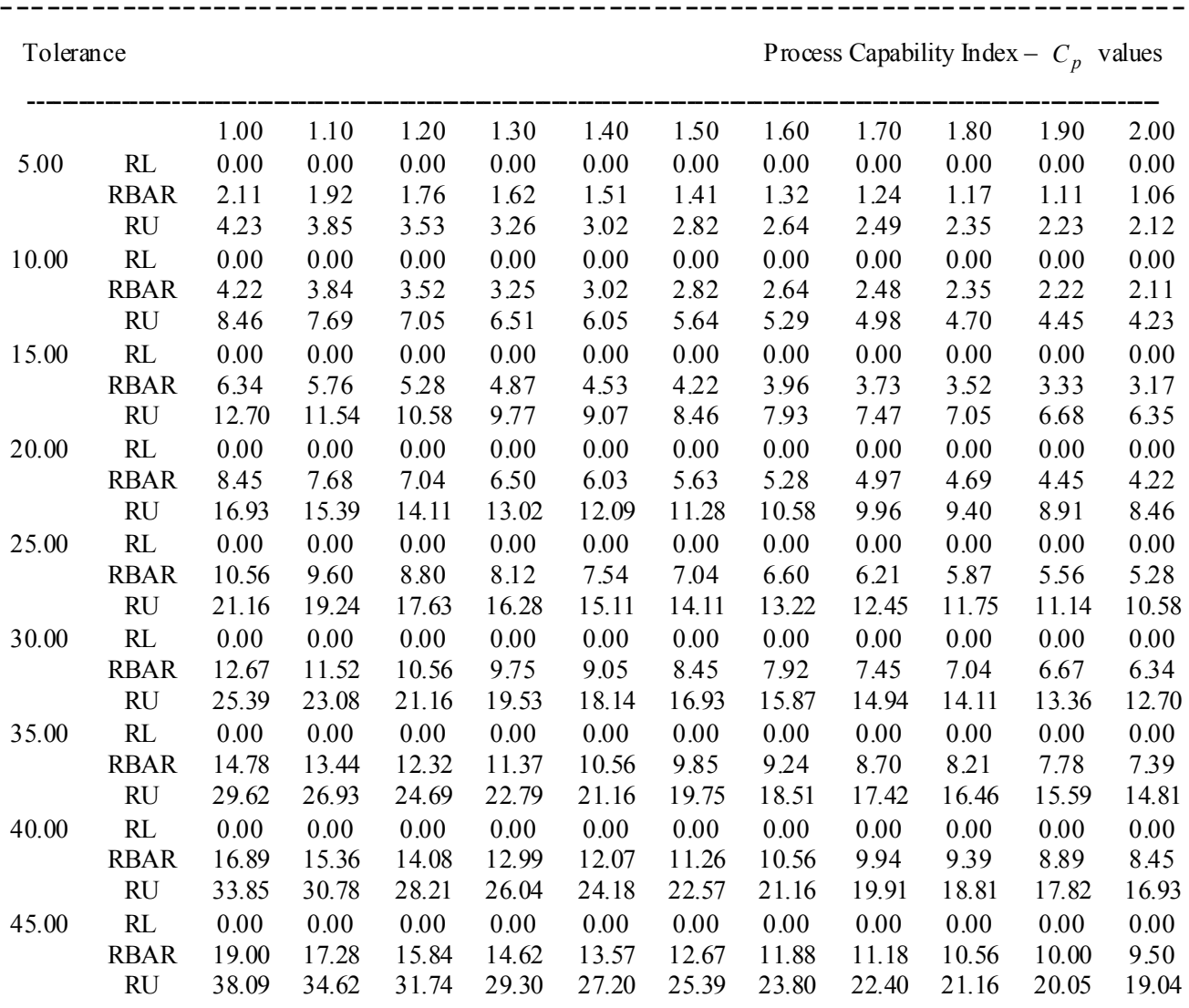




\begin{tabular}{|c|c|c|c|c|c|c|c|c|c|c|c|c|}
\hline \multirow[t]{3}{*}{50.00} & $\mathrm{RL}$ & 0.00 & 0.00 & 0.00 & 0.00 & 0.00 & 0.00 & 0.00 & 0.00 & 0.00 & 0.00 & 0.00 \\
\hline & RBAR & 21.12 & 19.20 & 17.60 & 16.24 & 15.08 & 14.08 & 13.20 & 12.42 & 11.73 & 11.11 & 10.56 \\
\hline & RU & 42.32 & 38.47 & 35.26 & 32.55 & 30.23 & 28.21 & 26.45 & 24.89 & 23.51 & 22.27 & 21.16 \\
\hline \multirow[t]{3}{*}{55.00} & RL & 0.00 & 0.00 & 0.00 & 0.00 & 0.00 & 0.00 & 0.00 & 0.00 & 0.00 & 0.00 & 0.00 \\
\hline & RBAR & 23.23 & 21.12 & 19.36 & 17.87 & 16.59 & 15.49 & 14.52 & 13.66 & 12.90 & 12.23 & 11.61 \\
\hline & RU & 46.55 & 42.32 & 38.79 & 35.81 & 33.25 & 31.03 & 29.09 & 27.38 & 25.86 & 24.50 & 23.27 \\
\hline \multirow[t]{3}{*}{60.00} & RL & 0.00 & 0.00 & 0.00 & 0.00 & 0.00 & 0.00 & 0.00 & 0.00 & 0.00 & 0.00 & 0.00 \\
\hline & RBAR & 25.34 & 23.04 & 21.12 & 19.49 & 18.10 & 16.89 & 15.84 & 14.91 & 14.08 & 13.34 & 12.67 \\
\hline & RU & 50.78 & 46.16 & 42.32 & 39.06 & 36.27 & 33.85 & 31.74 & 29.87 & 28.21 & 26.73 & 25.39 \\
\hline \multirow[t]{3}{*}{65.00} & RL & 0.00 & 0.00 & 0.00 & 0.00 & 0.00 & 0.00 & 0.00 & 0.00 & 0.00 & 0.00 & 0.00 \\
\hline & RBAR & 27.45 & 24.96 & 22.88 & 21.12 & 19.61 & 18.30 & 17.16 & 16.15 & 15.25 & 14.45 & 13.73 \\
\hline & RU & 55.01 & 50.01 & 45.84 & 42.32 & 39.30 & 36.68 & 34.38 & 32.36 & 30.56 & 28.95 & 27.51 \\
\hline \multirow[t]{3}{*}{70.00} & RL & 0.00 & 0.00 & 0.00 & 0.00 & 0.00 & 0.00 & 0.00 & 0.00 & 0.00 & 0.00 & 0.00 \\
\hline & RBAR & 29.56 & 26.88 & 24.64 & 22.74 & 21.12 & 19.71 & 18.48 & 17.39 & 16.42 & 15.56 & 14.78 \\
\hline & RU & 59.24 & 53.86 & 49.37 & 45.57 & 42.32 & 39.50 & 37.03 & 34.85 & 32.91 & 31.18 & 29.62 \\
\hline \multirow[t]{3}{*}{75.00} & RL & 0.00 & 0.00 & 0.00 & 0.00 & 0.00 & 0.00 & 0.00 & 0.00 & 0.00 & 0.00 & 0.00 \\
\hline & RBAR & 31.67 & 28.80 & 26.40 & 24.37 & 22.63 & 21.12 & 19.80 & 18.63 & 17.60 & 16.67 & 15.84 \\
\hline & RU & 63.48 & 57.71 & 52.90 & 48.83 & 45.34 & 42.32 & 39.67 & 37.34 & 35.26 & 33.41 & 31.74 \\
\hline \multirow[t]{3}{*}{80.00} & RL & 0.00 & 0.00 & 0.00 & 0.00 & 0.00 & 0.00 & 0.00 & 0.00 & 0.00 & 0.00 & 0.00 \\
\hline & RBAR & 33.79 & 30.72 & 28.16 & 25.99 & 24.13 & 22.52 & 21.12 & 19.87 & 18.77 & 17.78 & 16.89 \\
\hline & RU & 67.71 & 61.55 & 56.42 & 52.08 & 48.36 & 45.14 & 42.32 & 39.83 & 37.62 & 35.64 & 33.85 \\
\hline \multirow[t]{3}{*}{85.00} & RL & 0.00 & 0.00 & 0.00 & 0.00 & 0.00 & 0.00 & 0.00 & 0.00 & 0.00 & 0.00 & 0.00 \\
\hline & RBAR & 35.90 & 32.63 & 29.92 & 27.61 & 25.64 & 23.93 & 22.44 & 21.12 & 19.94 & 18.89 & 17.95 \\
\hline & RU & 71.94 & 65.40 & 59.95 & 55.34 & 51.39 & 47.96 & 44.96 & 42.32 & 39.97 & 37.86 & 35.97 \\
\hline \multirow[t]{3}{*}{90.00} & RL & 0.00 & 0.00 & 0.00 & 0.00 & 0.00 & 0.00 & 0.00 & 0.00 & 0.00 & 0.00 & 0.00 \\
\hline & RBAR & 38.01 & 34.55 & 31.68 & 29.24 & 27.15 & 25.34 & 23.76 & 22.36 & 21.12 & 20.01 & 19.00 \\
\hline & RU & 76.17 & 69.25 & 63.48 & 58.59 & 54.41 & 50.78 & 47.61 & 44.81 & 42.32 & 40.09 & 38.09 \\
\hline \multirow[t]{3}{*}{95.00} & $\mathrm{RL}$ & 0.00 & 0.00 & 0.00 & 0.00 & 0.00 & 0.00 & 0.00 & 0.00 & 0.00 & 0.00 & 0.00 \\
\hline & RBAR & 40.12 & 36.47 & 33.43 & 30.86 & 28.66 & 26.75 & 25.08 & 23.60 & 22.29 & 21.12 & 20.06 \\
\hline & RU & 80.40 & 73.09 & 67.00 & 61.85 & 57.43 & 53.60 & 50.25 & 47.30 & 44.67 & 42.32 & 40.20 \\
\hline \multirow[t]{3}{*}{100.00} & RL & 0.00 & 0.00 & 0.00 & 0.00 & 0.00 & 0.00 & 0.00 & 0.00 & 0.00 & 0.00 & 0.00 \\
\hline & RBAR & 42.23 & 38.39 & 35.19 & 32.49 & 30.17 & 28.16 & 26.40 & 24.84 & 23.46 & 22.23 & 21.12 \\
\hline & RU & 84.64 & 76.94 & 70.53 & 65.10 & 60.45 & 56.42 & 52.90 & 49.79 & 47.02 & 44.55 & 42.32 \\
\hline
\end{tabular}

Table 6. RL, RBAR and RU values for the given $C_{p}$ value and the Sample Size $\mathrm{n}=7$

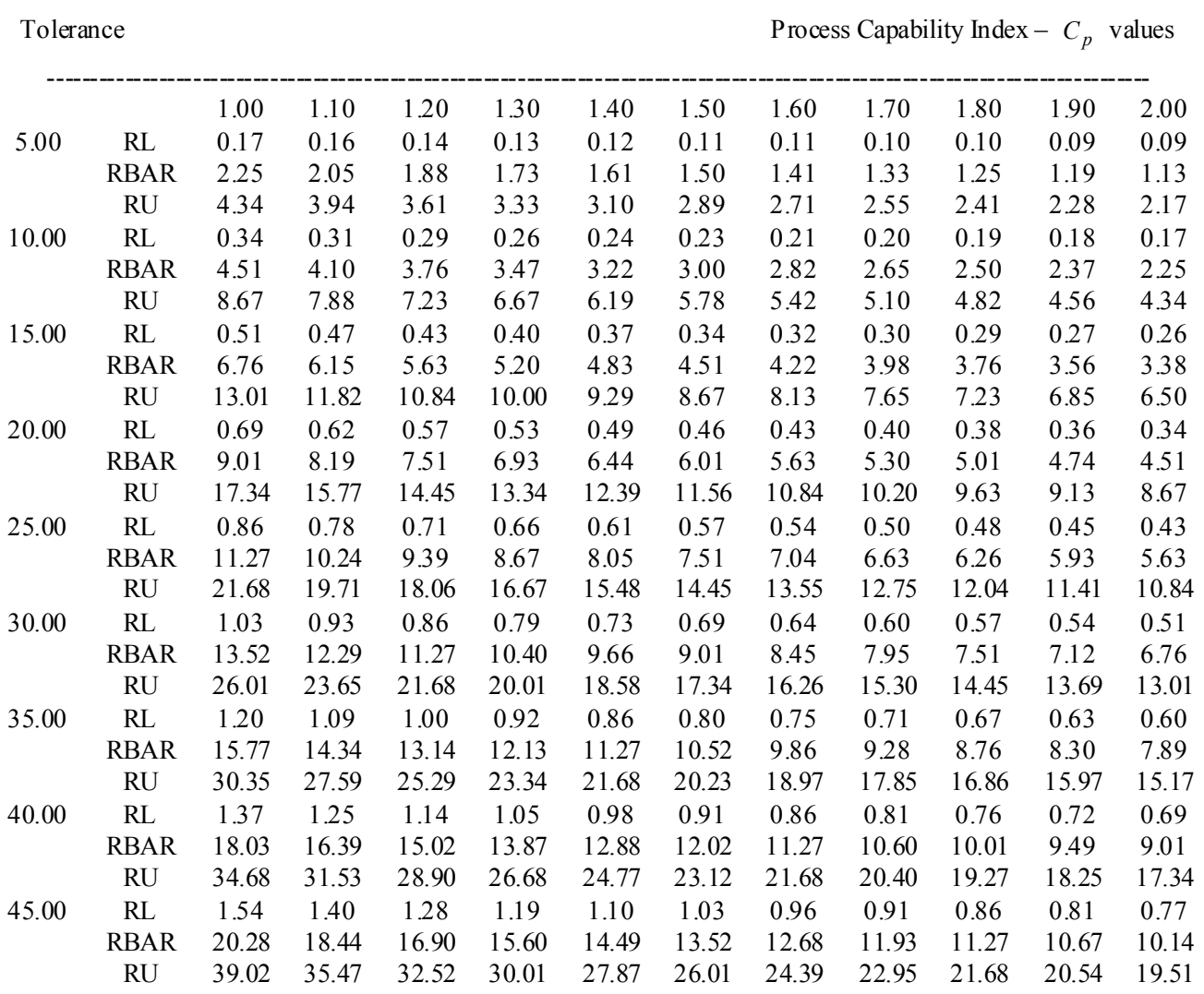




\begin{tabular}{|c|c|c|c|c|c|c|c|c|c|c|c|c|}
\hline \multirow[t]{3}{*}{50.00} & RL & 1.71 & 1.56 & 1.43 & 1.32 & 1.22 & 1.14 & 1.07 & 1.01 & 0.95 & 0.90 & 0.86 \\
\hline & RBAR & 22.53 & 20.48 & 18.78 & 17.33 & 16.10 & 15.02 & 14.08 & 13.25 & 12.52 & 11.86 & 11.27 \\
\hline & RU & 43.35 & 39.41 & 36.13 & 33.35 & 30.97 & 28.90 & 27.10 & 25.50 & 24.09 & 22.82 & 21.68 \\
\hline \multirow[t]{3}{*}{55.00} & RL & 1.88 & 1.71 & 1.57 & 1.45 & 1.35 & 1.26 & 1.18 & 1.11 & 1.05 & 0.99 & 0.94 \\
\hline & RBAR & 24.79 & 22.53 & 20.66 & 19.07 & 17.70 & 16.52 & 15.49 & 14.58 & 13.77 & 13.05 & 12.39 \\
\hline & RU & 47.69 & 43.35 & 39.74 & 36.68 & 34.06 & 31.79 & 29.81 & 28.05 & 26.49 & 25.10 & 23.84 \\
\hline \multirow[t]{3}{*}{60.00} & $\mathrm{RL}$ & 2.06 & 1.87 & 1.71 & 1.58 & 1.47 & 1.37 & 1.28 & 1.21 & 1.14 & 1.08 & 1.03 \\
\hline & RBAR & 27.04 & 24.58 & 22.53 & 20.80 & 19.31 & 18.03 & 16.90 & 15.91 & 15.02 & 14.23 & 13.52 \\
\hline & RU & 52.02 & 47.30 & 43.35 & 40.02 & 37.16 & 34.68 & 32.52 & 30.60 & 28.90 & 27.38 & 26.01 \\
\hline \multirow[t]{3}{*}{65.00} & RL & 2.23 & 2.02 & 1.86 & 1.71 & 1.59 & 1.48 & 1.39 & 1.31 & 1.24 & 1.17 & 1.11 \\
\hline & RBAR & 29.29 & 26.63 & 24.41 & 22.53 & 20.92 & 19.53 & 18.31 & 17.23 & 16.27 & 15.42 & 14.65 \\
\hline & RU & 56.36 & 51.24 & 46.97 & 43.35 & 40.26 & 37.57 & 35.23 & 33.15 & 31.31 & 29.66 & 28.18 \\
\hline \multirow[t]{3}{*}{70.00} & RL & 2.40 & 2.18 & 2.00 & 1.84 & 1.71 & 1.60 & 1.50 & 1.41 & 1.33 & 1.26 & 1.20 \\
\hline & RBAR & 31.55 & 28.68 & 26.29 & 24.27 & 22.53 & 21.03 & 19.72 & 18.56 & 17.53 & 16.60 & 15.77 \\
\hline & RU & 60.70 & 55.18 & 50.58 & 46.69 & 43.35 & 40.46 & 37.93 & 35.70 & 33.72 & 31.95 & 30.35 \\
\hline \multirow[t]{3}{*}{75.00} & $\mathrm{RL}$ & 2.57 & 2.34 & 2.14 & 1.98 & 1.83 & 1.71 & 1.61 & 1.51 & 1.43 & 1.35 & 1.28 \\
\hline & RBAR & 33.80 & 30.73 & 28.17 & 26.00 & 24.14 & 22.53 & 21.13 & 19.88 & 18.78 & 17.79 & 16.90 \\
\hline & RU & 65.03 & 59.12 & 54.19 & 50.02 & 46.45 & 43.35 & 40.64 & 38.25 & 36.13 & 34.23 & 32.52 \\
\hline \multirow[t]{3}{*}{80.00} & RL & 2.74 & 2.49 & 2.28 & 2.11 & 1.96 & 1.83 & 1.71 & 1.61 & 1.52 & 1.44 & 1.37 \\
\hline & RBAR & 36.05 & 32.78 & 30.04 & 27.73 & 25.75 & 24.04 & 22.53 & 21.21 & 20.03 & 18.98 & 18.03 \\
\hline & RU & 69.37 & 63.06 & 57.81 & 53.36 & 49.55 & 46.24 & 43.35 & 40.80 & 38.54 & 36.51 & 34.68 \\
\hline \multirow[t]{3}{*}{85.00} & RL & 2.91 & 2.65 & 2.43 & 2.24 & 2.08 & 1.94 & 1.82 & 1.71 & 1.62 & 1.53 & 1.46 \\
\hline & RBAR & 38.31 & 34.82 & 31.92 & 29.47 & 27.36 & 25.54 & 23.94 & 22.53 & 21.28 & 20.16 & 19.15 \\
\hline & RU & 73.70 & 67.00 & 61.42 & 56.69 & 52.64 & 49.13 & 46.06 & 43.35 & 40.95 & 38.79 & 36.85 \\
\hline \multirow[t]{3}{*}{90.00} & RL & 3.08 & 2.80 & 2.57 & 2.37 & 2.20 & 2.06 & 1.93 & 1.81 & 1.71 & 1.62 & 1.54 \\
\hline & RBAR & 40.56 & 36.87 & 33.80 & 31.20 & 28.97 & 27.04 & 25.35 & 23.86 & 22.53 & 21.35 & 20.28 \\
\hline & RU & 78.04 & 70.94 & 65.03 & 60.03 & 55.74 & 52.02 & 48.77 & 45.90 & 43.35 & 41.07 & 39.02 \\
\hline \multirow[t]{3}{*}{95.00} & RL & 3.25 & 2.96 & 2.71 & 2.50 & 2.32 & 2.17 & 2.03 & 1.91 & 1.81 & 1.71 & 1.63 \\
\hline & RBAR & 42.81 & 38.92 & 35.68 & 32.93 & 30.58 & 28.54 & 26.76 & 25.18 & 23.79 & 22.53 & 21.41 \\
\hline & RU & 82.37 & 74.88 & 68.64 & 63.36 & 58.84 & 54.92 & 51.48 & 48.45 & 45.76 & 43.35 & 41.19 \\
\hline \multirow[t]{3}{*}{100.00} & RL & 3.43 & 3.11 & 2.85 & 2.63 & 2.45 & 2.28 & 2.14 & 2.01 & 1.90 & 1.80 & 1.71 \\
\hline & RBAR & 45.07 & 40.97 & 37.56 & 34.67 & 32.19 & 30.04 & 28.17 & 26.51 & 25.04 & 23.72 & 22.53 \\
\hline & RU & 86.71 & 78.83 & 72.26 & 66.70 & 61.93 & 57.81 & 54.19 & 51.00 & 48.17 & 45.64 & 43.35 \\
\hline
\end{tabular}

Table 7. RL, RBAR and RU values for the given $C_{p}$ value and the Sample Size $\mathrm{n}=8$

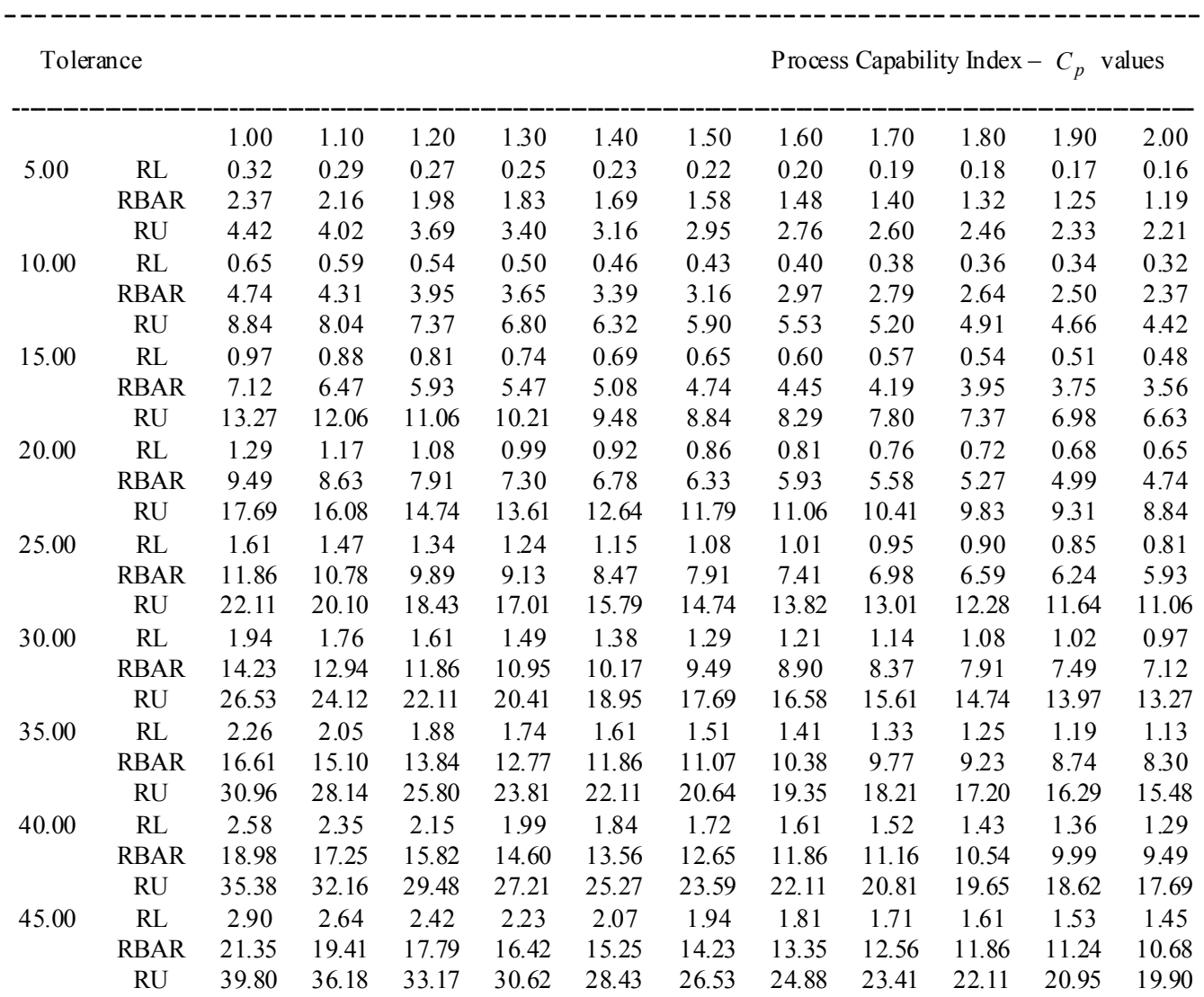




\begin{tabular}{|c|c|c|c|c|c|c|c|c|c|c|c|c|}
\hline \multirow[t]{3}{*}{50.00} & $\mathrm{RL}$ & 3.23 & 2.93 & 2.69 & 2.48 & 2.30 & 2.15 & 2.02 & 1.90 & 1.79 & 1.70 & 1.61 \\
\hline & RBAR & 23.72 & 21.57 & 19.77 & 18.25 & 16.95 & 15.82 & 14.83 & 13.96 & 13.18 & 12.49 & 11.86 \\
\hline & RU & 44.22 & 40.20 & 36.85 & 34.02 & 31.59 & 29.48 & 27.64 & 26.01 & 24.57 & 23.28 & 22.11 \\
\hline \multirow[t]{3}{*}{55.00} & RL & 3.55 & 3.23 & 2.96 & 2.73 & 2.54 & 2.37 & 2.22 & 2.09 & 1.97 & 1.87 & 1.77 \\
\hline & RBAR & 26.10 & 23.72 & 21.75 & 20.08 & 18.64 & 17.40 & 16.31 & 15.35 & 14.50 & 13.74 & 13.05 \\
\hline & RU & 48.65 & 44.22 & 40.54 & 37.42 & 34.75 & 32.43 & 30.40 & 28.62 & 27.03 & 25.60 & 24.32 \\
\hline \multirow[t]{3}{*}{60.00} & $\mathrm{RL}$ & 3.87 & 3.52 & 3.23 & 2.98 & 2.77 & 2.58 & 2.42 & 2.28 & 2.15 & 2.04 & 1.94 \\
\hline & RBAR & 28.47 & 25.88 & 23.73 & 21.90 & 20.34 & 18.98 & 17.79 & 16.75 & 15.82 & 14.98 & 14.23 \\
\hline & RU & 53.07 & 48.24 & 44.22 & 40.82 & 37.91 & 35.38 & 33.17 & 31.22 & 29.48 & 27.93 & 26.53 \\
\hline \multirow[t]{3}{*}{65.00} & $\mathrm{RL}$ & 4.19 & 3.81 & 3.50 & 3.23 & 3.00 & 2.80 & 2.62 & 2.47 & 2.33 & 2.21 & 2.10 \\
\hline & RBAR & 30.84 & 28.04 & 25.70 & 23.73 & 22.03 & 20.56 & 19.28 & 18.14 & 17.13 & 16.23 & 15.42 \\
\hline & RU & 57.49 & 52.26 & 47.91 & 44.22 & 41.06 & 38.33 & 35.93 & 33.82 & 31.94 & 30.26 & 28.75 \\
\hline \multirow[t]{3}{*}{70.00} & RL & 4.52 & 4.11 & 3.76 & 3.47 & 3.23 & 3.01 & 2.82 & 2.66 & 2.51 & 2.38 & 2.26 \\
\hline & RBAR & 33.22 & 30.20 & 27.68 & 25.55 & 23.73 & 22.14 & 20.76 & 19.54 & 18.45 & 17.48 & 16.61 \\
\hline & RU & 61.91 & 56.28 & 51.59 & 47.63 & 44.22 & 41.28 & 38.70 & 36.42 & 34.40 & 32.59 & 30.96 \\
\hline \multirow[t]{3}{*}{75.00} & $\mathrm{RL}$ & 4.84 & 4.40 & 4.03 & 3.72 & 3.46 & 3.23 & 3.02 & 2.85 & 2.69 & 2.55 & 2.42 \\
\hline & RBAR & 35.59 & 32.35 & 29.66 & 27.38 & 25.42 & 23.72 & 22.24 & 20.93 & 19.77 & 18.73 & 17.79 \\
\hline & RU & 66.34 & 60.30 & 55.28 & 51.03 & 47.38 & 44.22 & 41.46 & 39.02 & 36.85 & 34.91 & 33.17 \\
\hline \multirow[t]{3}{*}{80.00} & $\mathrm{RL}$ & 5.16 & 4.69 & 4.30 & 3.97 & 3.69 & 3.44 & 3.23 & 3.04 & 2.87 & 2.72 & 2.58 \\
\hline & RBAR & 37.96 & 34.51 & 31.63 & 29.20 & 27.11 & 25.31 & 23.72 & 22.33 & 21.09 & 19.98 & 18.98 \\
\hline & RU & 70.76 & 64.32 & 58.96 & 54.43 & 50.54 & 47.17 & 44.22 & 41.62 & 39.31 & 37.24 & 35.38 \\
\hline \multirow[t]{3}{*}{85.00} & RL & 5.49 & 4.99 & 4.57 & 4.22 & 3.92 & 3.66 & 3.43 & 3.23 & 3.05 & 2.89 & 2.74 \\
\hline & RBAR & 40.33 & 36.67 & 33.61 & 31.02 & 28.81 & 26.89 & 25.21 & 23.72 & 22.41 & 21.23 & 20.17 \\
\hline & RU & 75.18 & 68.35 & 62.65 & 57.83 & 53.70 & 50.12 & 46.99 & 44.22 & 41.77 & 39.57 & 37.59 \\
\hline \multirow[t]{3}{*}{90.00} & $\mathrm{RL}$ & 5.81 & 5.28 & 4.84 & 4.47 & 4.15 & 3.87 & 3.63 & 3.42 & 3.23 & 3.06 & 2.90 \\
\hline & RBAR & 42.70 & 38.82 & 35.59 & 32.85 & 30.50 & 28.47 & 26.69 & 25.12 & 23.73 & 22.48 & 21.35 \\
\hline & RU & 79.60 & 72.37 & 66.34 & 61.23 & 56.86 & 53.07 & 49.75 & 46.82 & 44.22 & 41.90 & 39.80 \\
\hline \multirow[t]{3}{*}{95.00} & RL & 6.13 & 5.57 & 5.11 & 4.72 & 4.38 & 4.09 & 3.83 & 3.61 & 3.41 & 3.23 & 3.07 \\
\hline & RBAR & 45.08 & 40.98 & 37.56 & 34.67 & 32.20 & 30.05 & 28.17 & 26.52 & 25.04 & 23.72 & 22.54 \\
\hline & RU & 84.02 & 76.39 & 70.02 & 64.63 & 60.02 & 56.02 & 52.52 & 49.43 & 46.68 & 44.22 & 42.01 \\
\hline \multirow[t]{3}{*}{100.00} & $\mathrm{RL}$ & 6.45 & 5.87 & 5.38 & 4.96 & 4.61 & 4.30 & 4.03 & 3.80 & 3.59 & 3.40 & 3.23 \\
\hline & RBAR & 47.45 & 43.14 & 39.54 & 36.50 & 33.89 & 31.63 & 29.66 & 27.91 & 26.36 & 24.97 & 23.72 \\
\hline & RU & 88.45 & 80.41 & 73.71 & 68.04 & 63.18 & 58.96 & 55.28 & 52.03 & 49.14 & 46.55 & 44.22 \\
\hline
\end{tabular}

Table 8. RL, RBAR and RU values for the given $C_{p}$ value and the Sample Size $\mathrm{n}=9$

\begin{tabular}{|c|c|c|c|c|c|c|c|c|c|c|c|c|}
\hline \multicolumn{8}{|c|}{ Tolerance } & \multicolumn{5}{|c|}{ Process Capability Index $-C_{p}$ values } \\
\hline & & 1.00 & 1.10 & 1.20 & 1.30 & 1.40 & 1.50 & 1.60 & 1.70 & 1.80 & 1.90 & 2.00 \\
\hline \multirow[t]{3}{*}{5.00} & RL & 0.46 & 0.41 & 0.38 & 0.35 & 0.33 & 0.30 & 0.28 & 0.27 & 0.25 & 0.24 & 0.23 \\
\hline & RBAR & 2.47 & 2.25 & 2.06 & 1.90 & 1.77 & 1.65 & 1.55 & 1.46 & 1.38 & 1.30 & 1.24 \\
\hline & RU & 4.49 & 4.09 & 3.75 & 3.46 & 3.21 & 3.00 & 2.81 & 2.64 & 2.50 & 2.37 & 2.25 \\
\hline \multirow[t]{3}{*}{10.00} & RL & 0.91 & 0.83 & 0.76 & 0.70 & 0.65 & 0.61 & 0.57 & 0.54 & 0.51 & 0.48 & 0.46 \\
\hline & RBAR & 4.95 & 4.50 & 4.13 & 3.81 & 3.54 & 3.30 & 3.09 & 2.91 & 2.75 & 2.61 & 2.47 \\
\hline & RU & 8.99 & 8.17 & 7.49 & 6.91 & 6.42 & 5.99 & 5.62 & 5.29 & 4.99 & 4.73 & 4.49 \\
\hline \multirow{3}{*}{15.00} & RL & 1.37 & 1.24 & 1.14 & 1.05 & 0.98 & 0.91 & 0.85 & 0.80 & 0.76 & 0.72 & 0.68 \\
\hline & RBAR & 7.43 & 6.75 & 6.19 & 5.71 & 5.30 & 4.95 & 4.64 & 4.37 & 4.13 & 3.91 & 3.71 \\
\hline & RU & 13.48 & 12.26 & 11.24 & 10.37 & 9.63 & 8.99 & 8.43 & 7.93 & 7.49 & 7.10 & 6.74 \\
\hline \multirow[t]{3}{*}{20.00} & RL & 1.82 & 1.66 & 1.52 & 1.40 & 1.30 & 1.21 & 1.14 & 1.07 & 1.01 & 0.96 & 0.91 \\
\hline & RBAR & 9.90 & 9.00 & 8.25 & 7.62 & 7.07 & 6.60 & 6.19 & 5.82 & 5.50 & 5.21 & 4.95 \\
\hline & RU & 17.98 & 16.34 & 14.98 & 13.83 & 12.84 & 11.99 & 11.24 & 10.58 & 9.99 & 9.46 & 8.99 \\
\hline \multirow[t]{3}{*}{25.00} & $\mathrm{RL}$ & 2.28 & 2.07 & 1.90 & 1.75 & 1.63 & 1.52 & 1.42 & 1.34 & 1.27 & 1.20 & 1.14 \\
\hline & RBAR & 12.38 & 11.25 & 10.31 & 9.52 & 8.84 & 8.25 & 7.73 & 7.28 & 6.88 & 6.51 & 6.19 \\
\hline & RU & 22.47 & 20.43 & 18.73 & 17.29 & 16.05 & 14.98 & 14.05 & 13.22 & 12.49 & 11.83 & 11.24 \\
\hline \multirow[t]{3}{*}{30.00} & RL & 2.73 & 2.48 & 2.28 & 2.10 & 1.95 & 1.82 & 1.71 & 1.61 & 1.52 & 1.44 & 1.37 \\
\hline & RBAR & 14.85 & 13.50 & 12.38 & 11.42 & 10.61 & 9.90 & 9.28 & 8.74 & 8.25 & 7.82 & 7.43 \\
\hline & RU & 26.97 & 24.52 & 22.47 & 20.74 & 19.26 & 17.98 & 16.85 & 15.86 & 14.98 & 14.19 & 13.48 \\
\hline \multirow[t]{3}{*}{35.00} & RL & 3.19 & 2.90 & 2.66 & 2.45 & 2.28 & 2.13 & 1.99 & 1.88 & 1.77 & 1.68 & 1.59 \\
\hline & RBAR & 17.33 & 15.75 & 14.44 & 13.33 & 12.38 & 11.55 & 10.83 & 10.19 & 9.63 & 9.12 & 8.66 \\
\hline & RU & 31.46 & 28.60 & 26.22 & 24.20 & 22.47 & 20.97 & 19.66 & 18.51 & 17.48 & 16.56 & 15.73 \\
\hline \multirow[t]{3}{*}{40.00} & $\mathrm{RL}$ & 3.64 & 3.31 & 3.04 & 2.80 & 2.60 & 2.43 & 2.28 & 2.14 & 2.02 & 1.92 & 1.82 \\
\hline & RBAR & 19.80 & 18.00 & 16.50 & 15.23 & 14.14 & 13.20 & 12.38 & 11.65 & 11.00 & 10.42 & 9.90 \\
\hline & RU & 35.96 & 32.69 & 29.96 & 27.66 & 25.68 & 23.97 & 22.47 & 21.15 & 19.98 & 18.92 & 17.98 \\
\hline \multirow[t]{2}{*}{45.00} & RL & 4.10 & 3.73 & 3.42 & 3.15 & 2.93 & 2.73 & 2.56 & 2.41 & 2.28 & 2.16 & 2.05 \\
\hline & RBAR & 22.27 & 20.25 & 18.56 & 17.13 & 15.91 & 14.85 & 13.92 & 13.10 & 12.38 & 11.72 & 11.14 \\
\hline
\end{tabular}




\begin{tabular}{|c|c|c|c|c|c|c|c|c|c|c|c|c|}
\hline & RU & 40.45 & 36.77 & 33.71 & 31.12 & 28.89 & 26.97 & 25.28 & 23.79 & 22.47 & 21.29 & 20.23 \\
\hline \multirow[t]{3}{*}{50.00} & $\mathrm{RL}$ & 4.55 & 4.14 & 3.80 & 3.50 & 3.25 & 3.04 & 2.85 & 2.68 & 2.53 & 2.40 & 2.28 \\
\hline & RBAR & 24.75 & 22.50 & 20.63 & 19.04 & 17.68 & 16.50 & 15.47 & 14.56 & 13.75 & 13.03 & 12.38 \\
\hline & RU & 44.95 & 40.86 & 37.46 & 34.57 & 32.10 & 29.96 & 28.09 & 26.44 & 24.97 & 23.66 & 22.47 \\
\hline \multirow[t]{3}{*}{55.00} & RL & 5.01 & 4.55 & 4.17 & 3.85 & 3.58 & 3.34 & 3.13 & 2.95 & 2.78 & 2.64 & 2.50 \\
\hline & RBAR & 27.23 & 24.75 & 22.69 & 20.94 & 19.45 & 18.15 & 17.02 & 16.01 & 15.13 & 14.33 & 13.61 \\
\hline & RU & 49.44 & 44.95 & 41.20 & 38.03 & 35.31 & 32.96 & 30.90 & 29.08 & 27.47 & 26.02 & 24.72 \\
\hline \multirow[t]{3}{*}{60.00} & $\mathrm{RL}$ & 5.46 & 4.97 & 4.55 & 4.20 & 3.90 & 3.64 & 3.42 & 3.21 & 3.04 & 2.88 & 2.73 \\
\hline & RBAR & 29.70 & 27.00 & 24.75 & 22.85 & 21.21 & 19.80 & 18.56 & 17.47 & 16.50 & 15.63 & 14.85 \\
\hline & RU & 53.94 & 49.03 & 44.95 & 41.49 & 38.53 & 35.96 & 33.71 & 31.73 & 29.96 & 28.39 & 26.97 \\
\hline \multirow{3}{*}{65.00} & RL & 5.92 & 5.38 & 4.93 & 4.55 & 4.23 & 3.95 & 3.70 & 3.48 & 3.29 & 3.12 & 2.96 \\
\hline & RBAR & 32.17 & 29.25 & 26.81 & 24.75 & 22.98 & 21.45 & 20.11 & 18.93 & 17.88 & 16.93 & 16.09 \\
\hline & RU & 58.43 & 53.12 & 48.69 & 44.95 & 41.74 & 38.95 & 36.52 & 34.37 & 32.46 & 30.75 & 29.21 \\
\hline \multirow[t]{3}{*}{70.00} & RL & 6.38 & 5.80 & 5.31 & 4.90 & 4.55 & 4.25 & 3.98 & 3.75 & 3.54 & 3.36 & 3.19 \\
\hline & RBAR & 34.65 & 31.50 & 28.88 & 26.65 & 24.75 & 23.10 & 21.66 & 20.38 & 19.25 & 18.24 & 17.33 \\
\hline & RU & 62.92 & 57.20 & 52.44 & 48.40 & 44.95 & 41.95 & 39.33 & 37.01 & 34.96 & 33.12 & 31.46 \\
\hline \multirow[t]{3}{*}{75.00} & $\mathrm{RL}$ & 6.83 & 6.21 & 5.69 & 5.25 & 4.88 & 4.55 & 4.27 & 4.02 & 3.80 & 3.60 & 3.42 \\
\hline & RBAR & 37.13 & 33.75 & 30.94 & 28.56 & 26.52 & 24.75 & 23.20 & 21.84 & 20.63 & 19.54 & 18.56 \\
\hline & RU & 67.42 & 61.29 & 56.18 & 51.86 & 48.16 & 44.95 & 42.14 & 39.66 & 37.45 & 35.48 & 33.71 \\
\hline \multirow[t]{3}{*}{80.00} & $\mathrm{RL}$ & 7.29 & 6.62 & 6.07 & 5.60 & 5.20 & 4.86 & 4.55 & 4.29 & 4.05 & 3.83 & 3.64 \\
\hline & RBAR & 39.60 & 36.00 & 33.00 & 30.46 & 28.29 & 26.40 & 24.75 & 23.29 & 22.00 & 20.84 & 19.80 \\
\hline & RU & 71.91 & 65.38 & 59.93 & 55.32 & 51.37 & 47.94 & 44.95 & 42.30 & 39.95 & 37.85 & 35.96 \\
\hline \multirow[t]{3}{*}{85.00} & $\mathrm{RL}$ & 7.74 & 7.04 & 6.45 & 5.96 & 5.53 & 5.16 & 4.84 & 4.55 & 4.30 & 4.07 & 3.87 \\
\hline & RBAR & 42.08 & 38.25 & 35.06 & 32.37 & 30.05 & 28.05 & 26.30 & 24.75 & 23.38 & 22.14 & 21.04 \\
\hline & RU & 76.41 & 69.46 & 63.67 & 58.78 & 54.58 & 50.94 & 47.76 & 44.95 & 42.45 & 40.21 & 38.20 \\
\hline \multirow[t]{3}{*}{90.00} & RL & 8.20 & 7.45 & 6.83 & 6.31 & 5.86 & 5.46 & 5.12 & 4.82 & 4.55 & 4.31 & 4.10 \\
\hline & RBAR & 44.55 & 40.50 & 37.13 & 34.27 & 31.82 & 29.70 & 27.84 & 26.21 & 24.75 & 23.45 & 22.27 \\
\hline & RU & 80.90 & 73.55 & 67.42 & 62.23 & 57.79 & 53.94 & 50.56 & 47.59 & 44.95 & 42.58 & 40.45 \\
\hline \multirow[t]{3}{*}{95.00} & RL & 8.65 & 7.87 & 7.21 & 6.66 & 6.18 & 5.77 & 5.41 & 5.09 & 4.81 & 4.55 & 4.33 \\
\hline & RBAR & 47.03 & 42.75 & 39.19 & 36.17 & 33.59 & 31.35 & 29.39 & 27.66 & 26.13 & 24.75 & 23.51 \\
\hline & RU & 85.40 & 77.63 & 71.16 & 65.69 & 61.00 & 56.93 & 53.37 & 50.23 & 47.44 & 44.95 & 42.70 \\
\hline \multirow[t]{3}{*}{100.00} & $\mathrm{RL}$ & 9.11 & 8.28 & 7.59 & 7.01 & 6.51 & 6.07 & 5.69 & 5.36 & 5.06 & 4.79 & 4.55 \\
\hline & RBAR & 49.50 & 45.00 & 41.25 & 38.08 & 35.36 & 33.00 & 30.94 & 29.12 & 27.50 & 26.05 & 24.75 \\
\hline & RU & 89.89 & 81.72 & 74.91 & 69.15 & 64.21 & 59.93 & 56.18 & 52.88 & 49.94 & 47.31 & 44.95 \\
\hline
\end{tabular}

Table 9. RL, RBAR and RU values for the given $C_{p}$ value and the Sample Size n=10

Tolerance

Process Capability Index $-C_{p}$ values

\begin{tabular}{|c|c|c|c|c|c|c|c|c|c|c|c|c|}
\hline & & 1.00 & 1.10 & 1.20 & 1.30 & 1.40 & 1.50 & 1.60 & 1.70 & 1.80 & 1.90 & \\
\hline & RL & & 0.52 & & & & & & & & & 0.29 \\
\hline \multirow[t]{3}{*}{5.00} & RBAR & & & & 1.97 & & & & & & & 1.28 \\
\hline & & & & & & & & & & & & 2.28 \\
\hline & & & & & & & & & & & & 0.57 \\
\hline \multirow[t]{3}{*}{10.00} & RBAR & 5.1 & & & 3.9 & & & & & & & 2.57 \\
\hline & & & & & & & & & & & & 4.56 \\
\hline & $\mathrm{RL}$ & & & & & & & & & & & 0.86 \\
\hline \multirow[t]{3}{*}{15.00} & RBAR & 7.70 & & & & & & & & & & 3.85 \\
\hline & & & & & & & & & & & & 6.84 \\
\hline & RL & & & & & 1. & & & & & & 1.14 \\
\hline \multirow[t]{3}{*}{20.00} & RBAR & 10.26 & & & & & & & & & & 5.13 \\
\hline & & 18.23 & 16.57 & 15.19 & 14.02 & 13.02 & 12.15 & & & & & 9.12 \\
\hline & RL & 2.86 & 2.60 & 2.38 & 2.20 & 2.04 & 1.91 & 1.79 & 1.6 & & & 1.43 \\
\hline \multirow[t]{3}{*}{25.00} & RBAR & 12.83 & 11.66 & 10.69 & & & & & & & & 6.41 \\
\hline & RU & 22.79 & 20.72 & 18.99 & 17.53 & 16.28 & 15 & 14.24 & 13.41 & 12.66 & 11.99 & 11.40 \\
\hline & RL & 3.43 & & 2.86 & 2.64 & & 2.29 & & 2.02 & & 1.81 & 1.72 \\
\hline \multirow[t]{3}{*}{30.00} & RBAR & & & & & & & & & & & 7.70 \\
\hline & & & & & & & & & & & & 13.67 \\
\hline & & & & & & & & & & & & 2.00 \\
\hline \multirow[t]{3}{*}{35.00} & & & 16.3 & & & 12.83 & & & & & & 8.98 \\
\hline & & & 29. & & 24.5 & 22.7 & & & & & & 15.95 \\
\hline & RL & & & & 3.52 & 3.27 & & & & & & 2.29 \\
\hline \multirow[t]{2}{*}{40.00} & RBAR & 20.52 & 18.65 & 17.10 & 15.78 & 14.66 & 13.68 & 12.82 & 12.07 & 11.40 & 10.80 & 10.26 \\
\hline & RU & 36.46 & 33.15 & 30.39 & 28.05 & 26.05 & 24.31 & 22.79 & 21.45 & 20.26 & 19.19 & 18.23 \\
\hline 5.00 & RL & 5.15 & 4.68 & 4.29 & 3.96 & 3.68 & 3.43 & 3.22 & 3.03 & 2.86 & 2.71 & 2.57 \\
\hline
\end{tabular}




\begin{tabular}{|c|c|c|c|c|c|c|c|c|c|c|c|c|}
\hline & RBAR & 23.09 & 20.99 & 19.24 & 17.76 & 16.49 & 15.39 & 14.43 & 13.58 & 12.83 & 12.15 & 11.54 \\
\hline & RU & 41.02 & 37.29 & 34.19 & 31.56 & 29.30 & 27.35 & 25.64 & 24.13 & 22.79 & 21.59 & 20.51 \\
\hline & RL & 5.72 & 5.20 & 4.77 & 4.40 & 4.09 & 3.81 & 3.57 & 3.36 & 3.18 & 3.01 & 2.86 \\
\hline \multirow[t]{3}{*}{50.00} & RBAR & 25.65 & 23.32 & 21.38 & 19.73 & 18.32 & 17.10 & 16.03 & 15.09 & 14.25 & 13.50 & 12.83 \\
\hline & RU & 45.58 & 41.44 & 37.98 & 35.06 & 32.56 & 30.39 & 28.49 & 26.81 & 25.32 & 23.99 & 22.79 \\
\hline & RL & 6.29 & 5.72 & 5.24 & 4.84 & 4.49 & 4.19 & 3.93 & 3.70 & 3.50 & 3.31 & 3.15 \\
\hline \multirow[t]{3}{*}{55.00} & RBAR & 28.22 & 25.65 & 23.51 & 21.70 & 20.15 & 18.81 & 17.63 & 16.60 & 15.68 & 14.85 & 14.11 \\
\hline & RU & 50.14 & 45.58 & 41.78 & 38.57 & 35.81 & 33.43 & 31.34 & 29.49 & 27.85 & 26.39 & 25.07 \\
\hline & RL & 6.86 & 6.24 & 5.72 & 5.28 & 4.90 & 4.58 & 4.29 & 4.04 & 3.81 & 3.61 & 3.43 \\
\hline \multirow[t]{3}{*}{60.00} & RBAR & 30.78 & 27.98 & 25.65 & 23.68 & 21.99 & 20.52 & 19.24 & 18.11 & 17.10 & 16.20 & 15.39 \\
\hline & RU & 54.70 & 49.72 & 45.58 & 42.07 & 39.07 & 36.46 & 34.19 & 32.17 & 30.39 & 28.79 & 27.35 \\
\hline & RL & 7.44 & 6.76 & 6.20 & 5.72 & 5.31 & 4.96 & 4.65 & 4.37 & 4.13 & 3.91 & 3.72 \\
\hline \multirow[t]{3}{*}{65.00} & RBAR & 33.35 & 30.31 & 27.79 & 25.65 & 23.82 & 22.23 & 20.84 & 19.61 & 18.53 & 17.55 & 16.67 \\
\hline & $\mathrm{RU}$ & 59.25 & 53.87 & 49.38 & 45.58 & 42.32 & 39.50 & 37.03 & 34.86 & 32.92 & 31.19 & 29.63 \\
\hline & RL & 8.01 & 7.28 & 6.67 & 6.16 & 5.72 & 5.34 & 5.00 & 4.71 & 4.45 & 4.21 & 4.00 \\
\hline \multirow[t]{3}{*}{70.00} & RBAR & 35.91 & 32.65 & 29.93 & 27.62 & 25.65 & 23.94 & 22.44 & 21.12 & 19.95 & 18.90 & 17.95 \\
\hline & RU & 63.81 & 58.01 & 53.18 & 49.09 & 45.58 & 42.54 & 39.88 & 37.54 & 35.45 & 33.59 & 31.91 \\
\hline & RL & 8.58 & 7.80 & 7.15 & 6.60 & 6.13 & 5.72 & 5.36 & 5.05 & 4.77 & 4.52 & 4.29 \\
\hline \multirow[t]{3}{*}{75.00} & RBAR & 38.48 & 34.98 & 32.06 & 29.60 & 27.48 & 25.65 & 24.05 & 22.63 & 21.38 & 20.25 & 19.24 \\
\hline & RU & 68.37 & 62.15 & 56.98 & 52.59 & 48.84 & 45.58 & 42.73 & 40.22 & 37.98 & 35.98 & 34.19 \\
\hline & $\mathrm{RL}$ & 9.15 & 8.32 & 7.63 & 7.04 & 6.54 & 6.10 & 5.72 & 5.38 & 5.08 & 4.82 & 4.58 \\
\hline \multirow[t]{3}{*}{80.00} & RBAR & 41.04 & 37.31 & 34.20 & 31.57 & 29.31 & 27.36 & 25.65 & 24.14 & 22.80 & 21.60 & 20.52 \\
\hline & RU & 72.93 & 66.30 & 60.77 & 56.10 & 52.09 & 48.62 & 45.58 & 42.90 & 40.52 & 38.38 & 36.46 \\
\hline & $\mathrm{RL}$ & 9.72 & 8.84 & 8.10 & 7.48 & 6.95 & 6.48 & 6.08 & 5.72 & 5.40 & 5.12 & 4.86 \\
\hline \multirow[t]{3}{*}{85.00} & RBAR & 43.60 & 39.64 & 36.34 & 33.54 & 31.15 & 29.07 & 27.25 & 25.65 & 24.23 & 22.95 & 21.80 \\
\hline & RU & 77.49 & 70.44 & 64.57 & 59.60 & 55.35 & 51.66 & 48.43 & 45.58 & 43.05 & 40.78 & 38.74 \\
\hline & $\mathrm{RL}$ & 10.30 & 9.36 & 8.58 & 7.92 & 7.35 & 6.86 & 6.43 & 6.06 & 5.72 & 5.42 & 5.15 \\
\hline \multirow[t]{3}{*}{90.00} & RBAR & 46.17 & 41.97 & 38.48 & 35.52 & 32.98 & 30.78 & 28.86 & 27.16 & 25.65 & 24.30 & 23.09 \\
\hline & $\mathrm{RU}$ & 82.04 & 74.59 & 68.37 & 63.11 & 58.60 & 54.70 & 51.28 & 48.26 & 45.58 & 43.18 & 41.02 \\
\hline & RL & 10.87 & 9.88 & 9.06 & 8.36 & 7.76 & 7.25 & 6.79 & 6.39 & 6.04 & 5.72 & 5.43 \\
\hline \multirow[t]{3}{*}{95.00} & RBAR & 48.74 & 44.30 & 40.61 & 37.49 & 34.81 & 32.49 & 30.46 & 28.67 & 27.08 & 25.65 & 24.37 \\
\hline & RU & 86.60 & 78.73 & 72.17 & 66.62 & 61.86 & 57.73 & 54.13 & 50.94 & 48.11 & 45.58 & 43.30 \\
\hline & RL & 11.44 & 10.40 & 9.53 & 8.80 & 8.17 & 7.63 & 7.15 & 6.73 & 6.36 & 6.02 & 5.72 \\
\hline \multirow[t]{2}{*}{100.00} & RBAR & 51.30 & 46.64 & 42.75 & 39.46 & 36.64 & 34.20 & 32.06 & 30.18 & 28.50 & 27.00 & 25.65 \\
\hline & RU & 91.16 & 82.87 & 75.97 & 70.12 & 65.11 & 60.77 & 56.98 & 53.62 & 50.64 & 47.98 & 45.58 \\
\hline
\end{tabular}

Table 10. $C_{p k}$ values for the given $C_{p}$ and the DELTA* values

\begin{tabular}{|c|ccccccccccccc|}
\hline \multicolumn{1}{|c}{ Delta } & \multicolumn{10}{c|}{ Process Capability Index $C_{n}$ Values } \\
\hline & 1.00 & 1.10 & 1.20 & 1.30 & 1.40 & 1.50 & 1.60 & 1.70 & 1.80 & 1.90 & 2.00 \\
\cline { 2 - 11 } 0 & & & & & & & & & & & & \\
2 & 0.98 & 1.10 & 1.20 & 1.30 & 1.40 & 1.50 & 1.60 & 1.70 & 1.80 & 1.90 & 2.00 \\
4 & 0.96 & 1.06 & 1.18 & 1.27 & 1.37 & 1.47 & 1.57 & 1.67 & 1.76 & 1.86 & 1.96 \\
6 & 0.94 & 1.03 & 1.13 & 1.25 & 1.34 & 1.44 & 1.54 & 1.63 & 1.73 & 1.82 & 1.92 \\
8 & 0.92 & 1.01 & 1.10 & 1.20 & 1.32 & 1.41 & 1.50 & 1.60 & 1.69 & 1.79 & 1.88 \\
10 & 0.90 & 0.99 & 1.08 & 1.17 & 1.26 & 1.35 & 1.44 & 1.53 & 1.62 & 1.71 & 1.80 \\
& & & & & & & & & & & \\
12 & 0.88 & 0.97 & 1.06 & 1.14 & 1.23 & 1.32 & 1.41 & 1.50 & 1.58 & 1.67 & 1.76 \\
14 & 0.86 & 0.95 & 1.03 & 1.12 & 1.20 & 1.29 & 1.38 & 1.46 & 1.55 & 1.63 & 1.72 \\
16 & 0.84 & 0.92 & 1.01 & 1.09 & 1.18 & 1.26 & 1.34 & 1.43 & 1.51 & 1.60 & 1.68 \\
18 & 0.82 & 0.90 & 0.98 & 1.07 & 1.15 & 1.23 & 1.31 & 1.39 & 1.48 & 1.56 & 1.64 \\
20 & 0.80 & 0.88 & 0.96 & 1.04 & 1.12 & 1.20 & 1.28 & 1.36 & 1.44 & 1.52 & 1.60 \\
& & & & & & & & & & & \\
22 & 0.78 & 0.86 & 0.94 & 1.01 & 1.09 & 1.17 & 1.25 & 1.33 & 1.40 & 1.48 & 1.56 \\
24 & 0.76 & 0.84 & 0.91 & 0.99 & 1.06 & 1.14 & 1.22 & 1.29 & 1.37 & 1.44 & 1.52 \\
26 & 0.74 & 0.81 & 0.89 & 0.96 & 1.04 & 1.11 & 1.18 & 1.26 & 1.33 & 1.41 & 1.48 \\
28 & 0.72 & 0.79 & 0.86 & 0.94 & 1.01 & 1.08 & 1.15 & 1.22 & 1.30 & 1.37 & 1.44 \\
30 & 0.70 & 0.77 & 0.84 & 0.91 & 0.98 & 1.05 & 1.12 & 1.19 & 1.26 & 1.33 & 1.40 \\
& & & & & & & & & & & \\
32 & 0.68 & 0.75 & 0.82 & 0.88 & 0.95 & 1.02 & 1.09 & 1.16 & 1.22 & 1.29 & 1.36 \\
34 & 0.66 & 0.73 & 0.79 & 0.86 & 0.92 & 0.99 & 1.06 & 1.12 & 1.19 & 1.25 & 1.32 \\
36 & 0.64 & 0.70 & 0.77 & 0.83 & 0.90 & 0.96 & 1.02 & 1.09 & 1.15 & 1.22 & 1.28 \\
38 & 0.62 & 0.68 & 0.74 & 0.81 & 0.87 & 0.93 & 0.99 & 1.05 & 1.12 & 1.18 & 1.24 \\
40 & 0.60 & 0.66 & 0.72 & 0.78 & 0.84 & 0.90 & 0.96 & 1.02 & 1.08 & 1.14 & 1.20 \\
\hline
\end{tabular}




\begin{tabular}{|c|c|c|c|c|c|c|c|c|c|c|c|}
\hline 42 & 0.58 & 0.64 & 0.70 & 0.75 & 0.81 & 0.87 & 0.93 & 0.99 & 1.04 & 1.10 & 1.16 \\
\hline 44 & 0.56 & 0.62 & 0.67 & 0.73 & 0.78 & 0.84 & 0.90 & 0.95 & 1.01 & 1.06 & 1.12 \\
\hline 46 & 0.54 & 0.59 & 0.65 & 0.70 & 0.76 & 0.81 & 0.86 & 0.92 & 0.97 & 1.03 & 1.08 \\
\hline 48 & 0.52 & 0.57 & 0.62 & 0.68 & 0.73 & 0.78 & 0.83 & 0.88 & 0.94 & 0.99 & 1.04 \\
\hline 50 & 0.50 & 0.55 & 0.60 & 0.65 & 0.70 & 0.75 & 0.80 & 0.85 & 0.90 & 0.95 & 1.00 \\
\hline 52 & 0.48 & 0.53 & 0.58 & 0.62 & 0.67 & 0.72 & 0.77 & 0.82 & 0.86 & 0.91 & 0.96 \\
\hline 54 & 0.46 & 0.51 & 0.55 & 0.60 & 0.64 & 0.69 & 0.74 & 0.78 & 0.83 & 0.87 & 0.92 \\
\hline 56 & 0.44 & 0.48 & 0.53 & 0.57 & 0.62 & 0.66 & 0.70 & 0.75 & 0.79 & 0.84 & 0.88 \\
\hline 58 & 0.42 & 0.46 & 0.50 & 0.55 & 0.59 & 0.63 & 0.67 & 0.71 & 0.76 & 0.80 & 0.84 \\
\hline 60 & 0.40 & 0.44 & 0.48 & 0.52 & 0.56 & 0.60 & 0.64 & 0.68 & 0.72 & 0.76 & 0.80 \\
\hline 62 & 0.38 & 0.42 & 0.46 & 0.49 & 0.53 & 0.57 & 0.61 & 0.65 & 0.68 & 0.72 & 0.76 \\
\hline 64 & 0.36 & 0.40 & 0.43 & 0.47 & 0.50 & 0.54 & 0.58 & 0.61 & 0.65 & 0.68 & 0.72 \\
\hline 66 & 0.34 & 0.37 & 0.41 & 0.44 & 0.48 & 0.51 & 0.54 & 0.58 & 0.61 & 0.65 & 0.68 \\
\hline 68 & 0.32 & 0.35 & 0.38 & 0.42 & 0.45 & 0.48 & 0.51 & 0.54 & 0.58 & 0.61 & 0.64 \\
\hline 70 & 0.30 & 0.33 & 0.36 & 0.39 & 0.42 & 0.45 & 0.48 & 0.51 & 0.54 & 0.57 & 0.60 \\
\hline 72 & 0.28 & 0.31 & 0.34 & 0.36 & 0.39 & 0.42 & 0.45 & 0.48 & 0.50 & 0.53 & 0.56 \\
\hline 74 & 0.26 & 0.29 & 0.31 & 0.34 & 0.36 & 0.39 & 0.42 & 0.44 & 0.47 & 0.49 & 0.52 \\
\hline 76 & 0.24 & 0.26 & 0.29 & 0.31 & 0.34 & 0.36 & 0.38 & 0.41 & 0.43 & 0.46 & 0.48 \\
\hline 78 & 0.22 & 0.24 & 0.26 & 0.29 & 0.31 & 0.33 & 0.35 & 0.37 & 0.40 & 0.42 & 0.44 \\
\hline 80 & 0.20 & 0.22 & 0.24 & 0.26 & 0.28 & 0.30 & 0.32 & 0.34 & 0.36 & 0.38 & 0.40 \\
\hline 82 & 0.18 & 0.20 & 0.22 & 0.23 & 0.25 & 0.27 & 0.29 & 0.31 & 0.32 & 0.34 & 0.36 \\
\hline 84 & 0.16 & 0.18 & 0.19 & 0.21 & 0.22 & 0.24 & 0.26 & 0.27 & 0.29 & 0.30 & 0.32 \\
\hline 86 & 0.14 & 0.15 & 0.17 & 0.18 & 0.20 & 0.21 & 0.22 & 0.24 & 0.25 & 0.27 & 0.28 \\
\hline 88 & 0.12 & 0.13 & 0.14 & 0.16 & 0.17 & 0.18 & 0.19 & 0.20 & 0.22 & 0.23 & 0.24 \\
\hline 90 & 0.10 & 0.11 & 0.12 & 0.13 & 0.14 & 0.15 & 0.16 & 0.17 & 0.18 & 0.19 & 0.20 \\
\hline 92 & 0.08 & 0.09 & 0.10 & 0.10 & 0.11 & 0.12 & 0.13 & 0.14 & 0.14 & 0.15 & 0.16 \\
\hline 94 & 0.06 & 0.07 & 0.07 & 0.08 & 0.08 & 0.09 & 0.10 & 0.10 & 0.11 & 0.11 & 0.12 \\
\hline 96 & 0.04 & 0.04 & 0.05 & 0.05 & 0.06 & 0.06 & 0.06 & 0.07 & 0.07 & 0.08 & 0.08 \\
\hline 98 & 0.02 & 0.02 & 0.02 & 0.03 & 0.03 & 0.03 & 0.03 & 0.03 & 0.04 & 0.04 & 0.04 \\
\hline 100 & 0.00 & 0.00 & 0.00 & 0.00 & 0.00 & 0.00 & 0.00 & 0.00 & 0.00 & 0.00 & 0.00 \\
\hline
\end{tabular}

\section{REFERENCES}

[1] Balamurali, S, Bootstrap confidence limits for short run capability indices, Quality Engineering, 15(4), 643-648,2003.

[7] Franklin, L.A. and Wasserman, G.S, Bootstrap lower confidence limits for process capability indices, Journal of Quality Technology, 24(4), 196-210, 1992.

[8] Kane, V.E, Process capability indices, Journal of Quality Technology, 18, 41-52, Corrigenda, p.265, 1986.

[9] Kotz, S. and Johnson, N.L, Process capability indices - a review, 1992-2000, Journal of Quality Technology, 34(1), 2-19, 2002.

[2] Balamurali, S. and Kalyanasundaram, M, Bootstrap lower confidence limits for the process capability indices $\mathrm{Cp}, \mathrm{Cpk}$ and $\mathrm{Cpm}$, International Journal of Quality and Reliability Management, 19( 8/9), 1088-1097, 2002.

[3] Bissell, A.F, How reliable is your capability index?, Applied Statistics, 39(3), 331-340, 1990.

[4] Boyles, R.A, The Taguchi capability index, Journal of Quality Technology, 23(1), 17-26, 1991.

[5] Chan, L.K., Cheng, S. W. and Spring, F.A, A new measure of process capability: Cpm, Journal of Quality Technology, 30(3), 162-175, 1988.

[6] Chou, Y., Owen, D.B. and Borrego, S.A, Lower confidence limits on process capability indices, Journal of Quality Technology, 22(3),223-229,1990.

[10] Montgomery, D.C, Introduction to Statistical Quality Control, John Wiley, New York, 2004

[11] Subramani, J, Application of Sy stematic Sampling in Process Control, Statistics and Applications- Journal of Society of statistics, Computer and Applications, (New Series), Vol 2, No2, pp 7-17, 2004

[12] Subramani, J, Process Control in the Presence of Linear Trend, Model Assisted Statistical Applications, 5, 272-281, 2010

[13] Yum, B-J. and Kim, K-W, A bibliography of the literature on process capability indices: 2000-2009, Quality and Reliability Engin eering International, 27(3),251-268,2011 